\title{
Pd-Catalyzed Oxidative Heck Reaction of Grignard Reagents with Diaziridinone as Oxidant
}

\author{
Qipu Dai, Baoguo Zhao, Yihui Yang, and Yian Shi* \\ Department of Chemistry \\ Colorado State University \\ Fort Collins, CO 80523, United States \\ E-mail: Yian.Shi@colostate.edu
}

\section{Supporting Information}

\section{Table of Contents}

General methods

S-2

Representative procedures for oxidative Heck reaction (Table 2, entry 1)

S-2

Characterization data

S-3

NMR spectra

S-12 
General methods. All commercially available reagents were used without further purification. Column chromatography was performed on silica gel (200-400 mesh). ${ }^{1} \mathrm{H}$ NMR spectra were recorded on a 300 or $400 \mathrm{MHz}$ NMR spectrometer and ${ }^{13} \mathrm{C}$ NMR spectra were recorded on a 75 or $100 \mathrm{MHz}$ NMR spectrometer. IR spectra were recorded on a FT-IR spectrometer. Melting points were uncorrected. Di-t-butyldiaziridinone (1) was prepared by following the reported procedure (H. Du, B. Zhao, Y. Shi, Org. Synth. 2009, 86, 315).

\section{Representative procedure for oxidative Heck reaction of Grignard reagents (Table 2, entry}

1, 4a): To a $4.0 \mathrm{~mL}$ flame dried vial equipped with a magnetic stir bar was added $\mathrm{Pd}(\mathrm{OAc})_{2}$ (0.00089 $\mathrm{g}, 0.0040 \mathrm{mmol})$. The sealed vial was vacuumed and refilled with argon three times, followed by the addition of dry ether $(0.25 \mathrm{~mL})$, THF $(0.05 \mathrm{~mL})$, styrene (3a) $(0.0208 \mathrm{~g}, 0.20$ $\mathrm{mmol})$, and di-t-butyldiaziridinone (1) $(0.068 \mathrm{~g}, 0.40 \mathrm{mmol})$ via syringe. The vial was placed in an ice bath and Grignard reagent $2 \mathbf{a}\left(2.0 \mathrm{M}\right.$ in $\left.\mathrm{Et}_{2} \mathrm{O}\right)(0.30 \mathrm{~mL}, 0.60 \mathrm{mmol})$ was added dropwise in $5 \mathrm{~min}$. The ice bath was removed and the reaction mixture was vigorously stirred at $\mathrm{rt}$ overnight. To the reaction mixture was added hexane to precipitate urea and magnesium salts. The solid was removed by filtration. The filtrate was concentrated and purified by flash chromatography ( silica gel, hexanes/ether/DCM $=100: 1: 2)$ to give product $4 \mathbf{a}(0.028 \mathrm{~g}, 72 \%)$ as a colorless oil.

\section{Representative procedure for oxidative Heck reaction of Grignard reagents on 1 mmol}

Scale (Table 2, entry 1, 4a): To a $10 \mathrm{~mL}$ flame dried vial equipped with a magnetic stir bar was added $\mathrm{Pd}(\mathrm{OAc})_{2}(0.0045 \mathrm{~g}, 0.020 \mathrm{mmol})$. The sealed vial was vacuumed and refilled with argon three times, followed by the addition of dry ether $(1.25 \mathrm{~mL})$, THF $(0.25 \mathrm{~mL})$, styrene (3a) $(0.104$ g, $1.00 \mathrm{mmol})$, and di-t-butyldiaziridinone (1) $(0.34 \mathrm{~g}, 2.0 \mathrm{mmol})$ via syringe. The vial was placed in an ice bath and Grignard reagent 2a $\left(2.0 \mathrm{M}_{\text {in }} \mathrm{Et}_{2} \mathrm{O}\right)(1.50 \mathrm{~mL}, 3.00 \mathrm{mmol})$ was added dropwise in $5 \mathrm{~min}$. The ice bath was removed and the reaction mixture was vigorously stirred at rt overnight. To the reaction mixture was added hexane to precipitate urea and magnesium salts. The solid was removed by filtration. The filtrate was concentrated and purified by flash 
chromatography ( silica gel, hexanes/ether/DCM $=100: 1: 2)$ to give product $4 \mathbf{a}(0.132 \mathrm{~g}, 68 \%)$ as a colorless oil.

\section{Characterization data}

Table 2, entry 1<smiles>Cc1ccccc1/C=C/c1ccccc1</smiles>

Colorless oil (0.028 g, 72\%); IR (film) 1598, $1495 \mathrm{~cm}^{-1} ;{ }^{1} \mathrm{H}$ NMR (400 MHz, $\left.\mathrm{CDCl}_{3}\right) \delta 7.60(\mathrm{~d}$, $J=7.2 \mathrm{~Hz}, 1 \mathrm{H}), 7.53(\mathrm{~d}, J=7.6 \mathrm{~Hz}, 2 \mathrm{H}), 7.37(\mathrm{dd}, J=8.0,7.2 \mathrm{~Hz}, 2 \mathrm{H}), 7.34$ (d, $J=16.4 \mathrm{~Hz}$, 1H), 7.30-7.16 (m, 4H), $7.00(\mathrm{~d}, J=16.4 \mathrm{~Hz}, 1 \mathrm{H}), 2.44(\mathrm{~s}, 3 \mathrm{H}) ;{ }^{13} \mathrm{C} \mathrm{NMR}\left(100 \mathrm{MHz}, \mathrm{CDCl}_{3}\right) \delta$ $137.8,136.5,135.9,130.5,130.1,128.8,127.73,127.69,126.70,126.69,126.3,125.5,20.1$.

E. Alacid, C. Na'jera, J. Org. Chem. 2009, 74, 2321.

\section{Table 2, entry 2}

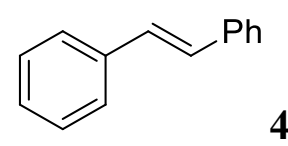

White solid (0.026 g, 72\%); m.p. 123-124 ${ }^{\circ} \mathrm{C}$; IR (film) 1598, 1495, 1452, $961 \mathrm{~cm}^{-1}$; ${ }^{1} \mathrm{H}$ NMR $\left(400 \mathrm{MHz}, \mathrm{CDCl}_{3}\right) \delta 7.52(\mathrm{~d}, J=7.6 \mathrm{~Hz}, 4 \mathrm{H}), 7.36(\mathrm{t}, J=7.6 \mathrm{~Hz}, 4 \mathrm{H}), 7.26(\mathrm{t}, J=7.6 \mathrm{~Hz}, 2 \mathrm{H})$, $7.11(\mathrm{~s}, 2 \mathrm{H}) ;{ }^{13} \mathrm{C} \mathrm{NMR}\left(100 \mathrm{MHz}, \mathrm{CDCl}_{3}\right) \delta 137.4,128.8,127.7,126.6$.

R. Wang, B. Twamley, J.M. Shreeve, J. Org. Chem. 2006, 71, 426.

\section{Table 2, entry 3}<smiles>Clc1ccc(/C=C/c2ccccc2)cc1</smiles>

White solid (0.027 g, 63\%); m.p. 122-123 ${ }^{\circ} \mathrm{C}$; IR (film) 1588, 1488, $1087 \mathrm{~cm}^{-1}$; ${ }^{1} \mathrm{H}$ NMR (400 $\left.\mathrm{MHz}, \mathrm{CDCl}_{3}\right) \delta 7.50(\mathrm{~d}, J=7.6 \mathrm{~Hz}, 2 \mathrm{H}), 7.43(\mathrm{~d}, J=8.4 \mathrm{~Hz}, 2 \mathrm{H}), 7.36(\mathrm{dd}, J=8.0,7.2 \mathrm{~Hz}, 2 \mathrm{H})$, $7.32(\mathrm{~d}, J=8.4 \mathrm{~Hz}, 2 \mathrm{H}), 7.27$ (t, $J=7.6 \mathrm{~Hz}, 1 \mathrm{H}), 7.08$ (d, $J=16.4 \mathrm{~Hz}, 1 \mathrm{H}), 7.04$ (d, $J=16.4 \mathrm{~Hz}$, 
$1 \mathrm{H}) ;{ }^{13} \mathrm{C} \mathrm{NMR}\left(75 \mathrm{MHz}, \mathrm{CDCl}_{3}\right) \delta 137.1,136.0,133.3,129.4,129.0,128.9,128.0,127.8,127.5$, 126.7 .

Z. Xi, B. Liu, W. Chen, J. Org. Chem. 2008, 73, 3954.

Table 2, entry 4

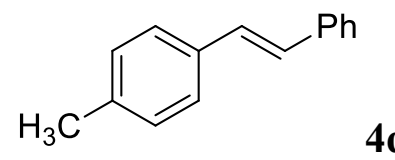

White solid (0.018 g, 46\%); m.p. 108-109 ${ }^{\circ} \mathrm{C}$; IR (film) 1593, 1509, $1448 \mathrm{~cm}^{-1}$; ${ }^{1} \mathrm{H}$ NMR (400 $\left.\mathrm{MHz}, \mathrm{CDCl}_{3}\right) \delta 7.50(\mathrm{~d}, J=8.0, \mathrm{~Hz}, 2 \mathrm{H}), \delta 7.41(\mathrm{~d}, J=8.0 \mathrm{~Hz}, 2 \mathrm{H}), \delta 7.35$ (t, $J=8.0,7.6, \mathrm{~Hz}$ 2H), $\delta 7.24$ (t, $J=7.6 \mathrm{~Hz}, 1 \mathrm{H}), \delta 7.17(\mathrm{~d}, J=8.0 \mathrm{~Hz}, 2 \mathrm{H}), 7.10$ (d, $J=16.4 \mathrm{~Hz}, 1 \mathrm{H}), 7.05$ (d, $J=$ $16.4 \mathrm{~Hz}, 1 \mathrm{H}), \delta 2.36(\mathrm{~s}, 3 \mathrm{H}) ;{ }^{13} \mathrm{C} \mathrm{NMR}\left(100 \mathrm{MHz}, \mathrm{CDCl}_{3}\right) \delta 137.7,134.7,129.5,128.79,128.77$, $127.8,127.5,126.6,126.5,21.4$.

R. Wang, B. Twamley, J.M. Shreeve, J. Org. Chem. 2006, 71, 426.

Table 2, entry 5<smiles>Cc1cccc(C)c1/C=C/c1ccccc1</smiles>

Colorless oil (0.030 g, 72\%); IR (film) 1594, $1465 \mathrm{~cm}^{-1} ;{ }^{1} \mathrm{H}$ NMR (400 MHz, $\left.\mathrm{CDCl}_{3}\right) \delta 7.53(\mathrm{~d}$, $J=7.6 \mathrm{~Hz}, 2 \mathrm{H}), 7.40$ (t, $J=7.6 \mathrm{~Hz}, 2 \mathrm{H}), 7.30$ (dd, $J=7.6,7.2 \mathrm{~Hz}, 1 \mathrm{H}), 7.14(\mathrm{~d}, J=16.8 \mathrm{~Hz}, 1 \mathrm{H})$, 7.12-7.07 (m, 3H), $6.63(\mathrm{~d}, J=16.8 \mathrm{~Hz}, 1 \mathrm{H}), 2.40(\mathrm{~s}, 6 \mathrm{H}) ;{ }^{13} \mathrm{C} \mathrm{NMR}\left(75 \mathrm{MHz}, \mathrm{CDCl}_{3}\right) \delta 137.7$, 137.1, 136.4, 134.1, 128.8, 128.0, 127.7, 127.0, 126.8, 126.4, 21.2.

H. Huang, H. Liu, H. Jiang, K. Chen, J. Org. Chem. 2008, 73, 6037.

Table 2, entry 6<smiles>Cc1cc(C)c(/C=C/c2ccccc2)c(C)c1</smiles> 
White solid (0.025 g, 56\%); m.p. 47-49 ${ }^{\circ} \mathrm{C}$; IR (film) 1596, 1448, $1375 \mathrm{~cm}^{-1} ;{ }^{1} \mathrm{H}$ NMR (400 $\left.\mathrm{MHz}, \mathrm{CDCl}_{3}\right) \delta 7.50(\mathrm{~d}, J=8.0 \mathrm{~Hz}, 2 \mathrm{H}), 7.37$ (t, $\left.J=7.6 \mathrm{~Hz}, 2 \mathrm{H}\right), 7.27$ (dd, $\left.J=7.6,7.2 \mathrm{~Hz}, 1 \mathrm{H}\right)$, $7.10(\mathrm{~d}, J=16.8 \mathrm{~Hz}, 1 \mathrm{H}), 6.91(\mathrm{~s}, 2 \mathrm{H}), 6.59(\mathrm{~d}, J=16.8 \mathrm{~Hz}, 1 \mathrm{H}), 2.34(\mathrm{~s}, 6 \mathrm{H}), 2.29(\mathrm{~s}, 3 \mathrm{H}) ;{ }^{13} \mathrm{C}$ $\operatorname{NMR}\left(75 \mathrm{MHz}, \mathrm{CDCl}_{3}\right) \delta 137.9,136.5,136.3,134.1,133.8,128.84,128.78,127.6,127.0,126.4$, 21.2, 21.1.

T. E. Barder, S.D. Walker, J. R. Martinelli, S. L. Buchwald, J. Am. Chem. Soc. 2005, 127, 4685.

Table 3, entry 1

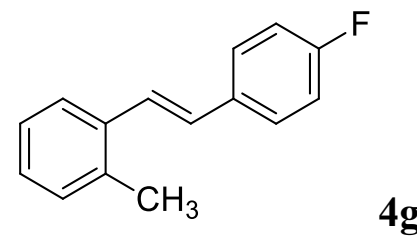

Colorless oil (0.029 g, 68\%); IR (film) 1600, 1507, 1459, $1231 \mathrm{~cm}^{-1} ;{ }^{1} \mathrm{H}$ NMR (300 MHz, $\left.\mathrm{CDCl}_{3}\right) \delta 7.63(\mathrm{~d}, J=6.9 \mathrm{~Hz}, 1 \mathrm{H}), 7.59-7.50(\mathrm{~m}, 2 \mathrm{H}), 7.31(\mathrm{~d}, J=16.2 \mathrm{~Hz}, 1 \mathrm{H}), 7.32-7.22(\mathrm{~m}$, 3H), 7.17-7.07 (m, 2H), $7.03(\mathrm{~d}, J=16.2 \mathrm{~Hz}, 1 \mathrm{H}), 2.49(\mathrm{~s}, 3 \mathrm{H}) ;{ }^{13} \mathrm{C} \mathrm{NMR}\left(75 \mathrm{MHz}, \mathrm{CDCl}_{3}\right) \delta$ $164.1,160.8,136.3,135.9,134.0,133.9,130.6,128.9,128.2,128.1,127.7,126.42,126.39$, 126.36, 125.4, 115.9, 115.6, 20.1; HRMS calcd for $\mathrm{C}_{15} \mathrm{H}_{13} \mathrm{~F}\left(\mathrm{M}^{+}\right)$: 212.1001; found: 212.0997 . Z. Chen, M. Luo, Y. Wen, G. Luo, L. Liu, Org. Lett. 2014, 16, 3020.

Table 3, entry 2

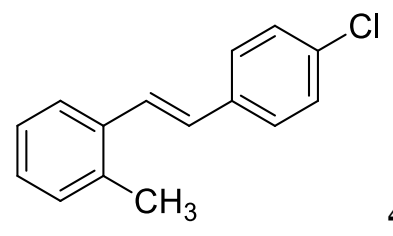

White solid (0.039 g, 86\%); m.p. $63-64{ }^{\circ} \mathrm{C}$; IR (film) 1489, 1457, $1086 \mathrm{~cm}^{-1} ;{ }^{1} \mathrm{H}$ NMR (400 $\left.\mathrm{MHz}, \mathrm{CDCl}_{3}\right) \delta 7.58(\mathrm{~d}, J=7.6 \mathrm{~Hz}, 1 \mathrm{H}), 7.45(\mathrm{~d}, J=8.4 \mathrm{~Hz}, 2 \mathrm{H}), 7.33(\mathrm{~d}, J=8.4 \mathrm{~Hz}, 2 \mathrm{H}), 7.31$ $(\mathrm{d}, J=16.4 \mathrm{~Hz}, 1 \mathrm{H}), 7.28-7.18(\mathrm{~m}, 3 \mathrm{H}), 6.95(\mathrm{~d}, J=16.4 \mathrm{~Hz}, 1 \mathrm{H}), 2.44(\mathrm{~s}, 3 \mathrm{H}) ;{ }^{13} \mathrm{C}$ NMR $(75$ $\left.\mathrm{MHz}, \mathrm{CDCl}_{3}\right) \delta 136.3,136.1,136.0,133.3,130.6,128.9,128.8,127.9,127.8,127.2,126.4$, 125.4, 20.1. HRMS calcd for $\mathrm{C}_{15} \mathrm{H}_{13} \mathrm{Cl}\left(\mathrm{M}^{+}\right)$: 228.0706; found: 228.0707 .

A. Bhunia, A. Studer, ACS Catal. 2018, 8, 1213. 
Table 3, entry 3

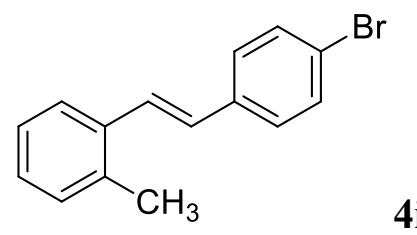

White solid (0.038 g, 70\%); m.p. 66-67 ${ }^{\circ} \mathrm{C}$; IR (film) 1487, 1457, $1069 \mathrm{~cm}^{-1} ;{ }^{1} \mathrm{H}$ NMR (400 $\left.\mathrm{MHz}, \mathrm{CDCl}_{3}\right) \delta 7.57(\mathrm{~d}, J=7.2 \mathrm{~Hz}, 1 \mathrm{H}), 7.48(\mathrm{~d}, J=8.4 \mathrm{~Hz}, 2 \mathrm{H}), 7.38(\mathrm{~d}, J=8.4 \mathrm{~Hz}, 2 \mathrm{H}), 7.32$ $(\mathrm{d}, J=16.0 \mathrm{~Hz}, 1 \mathrm{H}), 7.25-7.16(\mathrm{~m}, 3 \mathrm{H}), 6.92(\mathrm{~d}, J=16.0 \mathrm{~Hz}, 1 \mathrm{H}), 2.43(\mathrm{~s}, 3 \mathrm{H}) ;{ }^{13} \mathrm{C}$ NMR $(75$ $\left.\mathrm{MHz}, \mathrm{CDCl}_{3}\right) \delta 136.7,136.1,136.0,131.9,130.6,128.8,128.2,127.9,127.3,126.4,125.5$, 121.4, 20.1; HRMS calcd for $\mathrm{C}_{15} \mathrm{H}_{13} \mathrm{Br}\left(\mathrm{M}^{+}\right)$: 272.0201 ; found: 272.0199 .

A. Bhunia, A. Studer, ACS Catal. 2018, 8, 1213.

\section{Table 3, entry 4}<smiles>Cc1ccc(/C=C/c2ccccc2C)cc1</smiles>

Colorless oil (0.026 g, 63\%); IR (film) 1573, 1511, 1483, $1457 \mathrm{~cm}^{-1}$; ${ }^{1} \mathrm{H}$ NMR (400 MHz, $\left.\mathrm{CDCl}_{3}\right) \delta 7.58(\mathrm{~d}, J=7.2 \mathrm{~Hz}, 1 \mathrm{H}), 7.42(\mathrm{~d}, J=8.0 \mathrm{~Hz}, 2 \mathrm{H}), 7.29(\mathrm{~d}, J=16.0 \mathrm{~Hz}, 1 \mathrm{H}), 7.24-7.13$ $(\mathrm{m}, 5 \mathrm{H}), 6.98(\mathrm{~d}, J=16.0 \mathrm{~Hz}, 1 \mathrm{H}), 2.43(\mathrm{~s}, 3 \mathrm{H}), 2.37(\mathrm{~s}, 3 \mathrm{H}) ;{ }^{13} \mathrm{C} \mathrm{NMR}\left(75 \mathrm{MHz}, \mathrm{CDCl}_{3}\right) \delta$ 137.6, 136.7, 135.8, 135.0, 130.5, 130.0, 129.5, 127.5, 126.6, 126.3, 125.7, 125.4, 21.4, 20.1.

E. Shirakawa, X. Zhang, T. Hayashi Angew. Chem. Int. Ed. 2011, 50, 4671.

Table 3, entry 5<smiles>Cc1ccccc1/C=C/c1ccc(-c2ccccc2)cc1</smiles> 
White solid (0.035 g, 65\%); m.p. 108-109 ${ }^{\circ} \mathrm{C}$; IR (film) 1486, $1408 \mathrm{~cm}^{-1} ;{ }^{1} \mathrm{H}$ NMR (400 MHz, $\left.\mathrm{CDCl}_{3}\right) \delta 7.65-7.58(\mathrm{~m}, 7 \mathrm{H}), 7.45(\mathrm{t}, J=7.6 \mathrm{~Hz}, 2 \mathrm{H}), 7.38(\mathrm{~d}, J=16.4 \mathrm{~Hz}, 1 \mathrm{H}), 7.35(\mathrm{t}, J=7.2$ $\mathrm{Hz}, 1 \mathrm{H}), 7.35-7.16(\mathrm{~m}, 3 \mathrm{H}), 7.04(\mathrm{~d}, J=16.4 \mathrm{~Hz}, 1 \mathrm{H}), 2.45(\mathrm{~s}, 3 \mathrm{H}) ;{ }^{13} \mathrm{C} \mathrm{NMR}(100 \mathrm{MHz}$, $\left.\mathrm{CDCl}_{3}\right) \delta 140.8,140.5,136.9,136.5,136.0,130.6,129.6,129.0,127.7,127.51,127.47,127.13$, 127.07, 126.7, 126.4, 125.5, 20.1; HRMS calcd for $\mathrm{C}_{21} \mathrm{H}_{18}\left(\mathrm{M}^{+}\right)$: 270.1409; found: 270.1408 .

A. Bhunia, A. Studer, ACS Catal. 2018, 8, 1213.

Table 3, entry 6

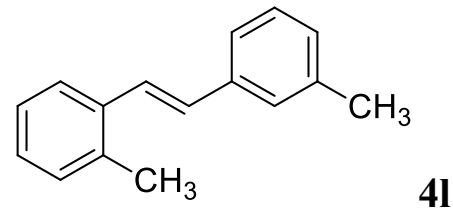

Colorless oil (0.029 g, 70\%); IR (film) 1602, 1489, $1378 \mathrm{~cm}^{-1} ;{ }^{1} \mathrm{H}$ NMR (400 MHz, $\left.\mathrm{CDCl}_{3}\right) \delta$ $7.60(\mathrm{~d}, J=7.2 \mathrm{~Hz}, 1 \mathrm{H}), 7.38-7.30(\mathrm{~m}, 3 \mathrm{H}), 7.27(\mathrm{t}, J=7.6 \mathrm{~Hz}, 1 \mathrm{H}), 7.24-7.15(\mathrm{~m}, 3 \mathrm{H}), 7.10(\mathrm{~d}$, $J=7.6 \mathrm{~Hz}, 1 \mathrm{H}), 6.99(\mathrm{~d}, J=16.4 \mathrm{~Hz}, 1 \mathrm{H}), 2.45(\mathrm{~s}, 3 \mathrm{H}), 2.40(\mathrm{~s}, 3 \mathrm{H}) ;{ }^{13} \mathrm{C} \mathrm{NMR}(100 \mathrm{MHz}$, $\left.\mathrm{CDCl}_{3}\right) \delta 138.4,137.8,136.6,135.9,130.5,130.3,128.7,128.6,127.6,127.4,126.5,126.3$, $125.5,123.9,21.6,20.1$.

A. Bhunia, A. Studer, ACS Catal. 2018, 8, 1213.

Table 3, entry 7

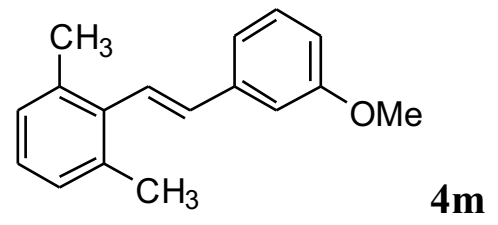

Pale yellow oil (0.034 g, 71\%); IR (film) 1595, 1578, 1464, $1270 \mathrm{~cm}^{-1}$; ${ }^{1} \mathrm{H}$ NMR (300 MHz, $\left.\mathrm{CDCl}_{3}\right) \delta 7.33(\mathrm{t}, J=7.8 \mathrm{~Hz}, 1 \mathrm{H}), 7.15(\mathrm{~d}, J=16.5 \mathrm{~Hz}, 1 \mathrm{H}) ; 7.19-7.05(\mathrm{~m}, 5 \mathrm{H}), 6.86(\mathrm{ddd}, J=$ 8.1, 2.4, 0.9 Hz, 1H), $6.62(\mathrm{~d}, J=16.5 \mathrm{~Hz}, 1 \mathrm{H}), 3.89(\mathrm{~s}, 3 \mathrm{H}), 2.41(\mathrm{~s}, 6 \mathrm{H}) ;{ }^{13} \mathrm{C} \mathrm{NMR}(75 \mathrm{MHz}$, $\left.\mathrm{CDCl}_{3}\right) \delta 160.0,139.2,136.9,136.3,134.0,129.8,128.0,127.4,126.9,119.1,113.1,111.9,55.4$ 21.2; HRMS calcd for $\mathrm{C}_{17} \mathrm{H}_{18} \mathrm{O}\left(\mathrm{M}^{+}\right)$: 238.1358; found: 238.1355. 
Table 3, entry 8

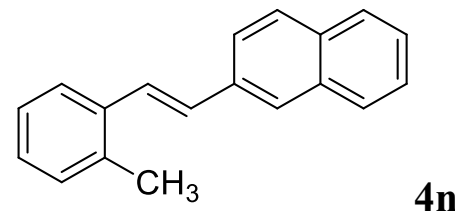

White solid (0.036 g, 74\%); m.p. 86-87 ${ }^{\circ} \mathrm{C}$; IR (film) 1595, $1460 \mathrm{~cm}^{-1}$; ${ }^{1} \mathrm{H}$ NMR (400 MHz, $\left.\mathrm{CDCl}_{3}\right) \delta$ 7.88-7.80 (m, 4H), $7.76(\mathrm{~d}, J=8.8 \mathrm{~Hz}, 1 \mathrm{H}), 7.66(\mathrm{~d}, J=7.2 \mathrm{~Hz}, 1 \mathrm{H}), 7.52-7.42(\mathrm{~m}$, 3H), 7.26-7.19 (m, 3H), $7.17(\mathrm{~d}, J=16.4 \mathrm{~Hz}, 1 \mathrm{H}), 2.48(\mathrm{~s}, 3 \mathrm{H}) ;{ }^{13} \mathrm{C} \mathrm{NMR}\left(75 \mathrm{MHz}, \mathrm{CDCl}_{3}\right) \delta$ 136.5, 136.0, 135.3, 133.8, 133.1, 130.6, 130.2, 128.4, 128.1, 127.84, 127.75, 126.9, 126.8, 126.5, $126.4,126.0,125.5,123.7,20.2$.

A. Bhunia, A. Studer, ACS Catal. 2018, 8, 1213.

Table 3, entry 9<smiles>Cc1cccc(C)c1/C=C/[Si](C)(C)C</smiles>

40

Colorless oil (0.037 g, 91\%); IR (film) 1487, 1457, $1399 \mathrm{~cm}^{-1} ;{ }^{1} \mathrm{H}$ NMR (400 MHz, $\left.\mathrm{CDCl}_{3}\right) \delta$ 7.10-6.96 (m, 3H), 6.87 (d, $J=19.6 \mathrm{~Hz}, 1 \mathrm{H}), 5.92$ (d, $J=19.6 \mathrm{~Hz}, 1 \mathrm{H}), 2.28$ (s, 6H), 0.17 (s, $9 \mathrm{H}) ;{ }^{13} \mathrm{C}$ NMR $\left(75 \mathrm{MHz}, \mathrm{CDCl}_{3}\right) \delta 142.5,139.6,136.2,135.4,127.9,126.6,20.9,-1.1$; HRMS calcd for $\mathrm{C}_{13} \mathrm{H}_{20} \mathrm{Si}\left(\mathrm{M}^{+}\right)$: 204.1334; found: 204.1331.

Table 3, entry 10

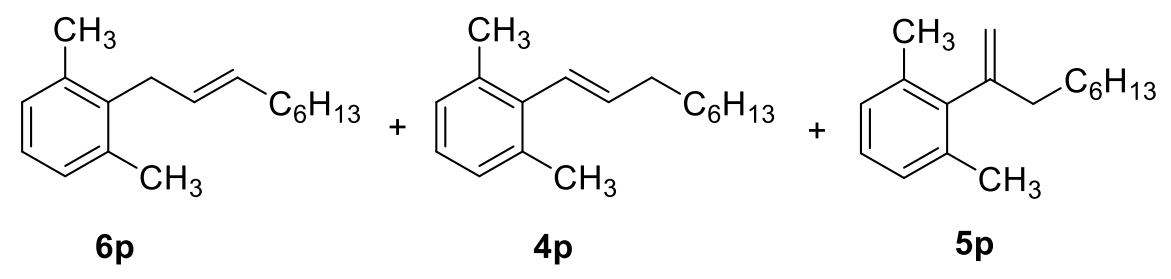

Colorless oil (0.022 g, 48\%); IR (film) 1465, $1377 \mathrm{~cm}^{-1} ;{ }^{1} \mathrm{H}$ NMR (400 MHz, $\left.\mathrm{CDCl}_{3}\right) \delta 7.15$ $6.95(\mathrm{~m}, 3 \mathrm{H}), 6.29(\mathrm{~d}, J=16.4 \mathrm{~Hz}, 0.11 \mathrm{H}$ for $4 \mathrm{p}), 5.65(\mathrm{dt}, J=16.4,6.8 \mathrm{~Hz}, 0.11 \mathrm{H}$ for $4 \mathrm{p}), 5.44$ (dt, $J=15.2,6.0 \mathrm{~Hz}, 0.89 \mathrm{H}$ for $\mathbf{6 p}$ and $\mathbf{5 p}), 5.36-5.22(\mathrm{~m}, 0.89 \mathrm{H}$ for $\mathbf{6 p}), 4.80(\mathrm{~s}, 0.08 \mathrm{H}$ for $\mathbf{5 p}$ ), 
$3.32(\mathrm{~d}, J=5.6 \mathrm{~Hz}, 1.62 \mathrm{H}$ for $\mathbf{6 p}), 2.63-2.15(\mathrm{~m}, 6.38 \mathrm{H}), 1.95(\mathrm{q}, J=6.8 \mathrm{~Hz}, 1.62 \mathrm{H}$ for $\mathbf{6 p})$,

1.51-1.18 (m, 8.38H), 0.93-0.80 (m, 3H); ${ }^{13} \mathrm{C} \mathrm{NMR}\left(100 \mathrm{MHz}, \mathrm{CDCl}_{3}\right) \delta 137.3,136.7,131.3$, $128.1,126.5,125.9,32.8,32.7,31.9,29.6,29.0,22.8,20.1,14.2$.

(1) Y. Nakao, S. Ebata, J. Chen, H. Imanaka, T. Hiyama Chem. Lett. 2007, 36, 606. (2) M. Thimmaiah, X. Zhang, S. Fang Tetrahedron Lett. 2008, 49, 5605. (3) R. Knorr, M. Knittl, C. Behringer, J. Ruhdorfer, P. Böhrer J. Org. Chem. 2017, 82, 2843.

\section{Table 3, entry 11}<smiles>C/C(=C\c1ccccc1)c1ccccc1C</smiles>

Colorless oil (0.020 g, 48\%); IR (film) 1599, 1489, $1442 \mathrm{~cm}^{-1} ;{ }^{1} \mathrm{H}$ NMR (400 MHz, $\left.\mathrm{CDCl}_{3}\right) \delta$ 7.43-7.34 (m, 4H), 7.28-7.15 (m, 5H), $6.38(\mathrm{~s}, 1 \mathrm{H}), 2.36(\mathrm{~s}, 3 \mathrm{H}), 2.19(\mathrm{~s}, 3 \mathrm{H}) ;{ }^{13} \mathrm{C}$ NMR $(75$ $\left.\mathrm{MHz}, \mathrm{CDCl}_{3}\right) \delta 145.9,139.2,138.2,134.9,130.3,129.3,129.1,128.3,128.2,126.9,126.6$, 125.8, 20.1, 20.0.

X. Xu, J. Chen, W. Gao, H. Wu, J. Ding, W. Su, Tetrahedron 2010, 66, 2433.

Table 3, entry 12

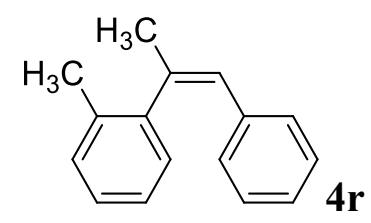

Colorless oil (0.024 g, 58\%); IR (film) 1598, 1488, $1439 \mathrm{~cm}^{-1} ;{ }^{1} \mathrm{H}$ NMR (400 MHz, $\left.\mathrm{CDCl}_{3}\right) \delta$ 7.25-7.15 (m, 3H), 7.12-7.00 (m, 4H), $6.83(\mathrm{~d}, J=8.0 \mathrm{~Hz}, 2 \mathrm{H}), 6.47(\mathrm{~s}, 1 \mathrm{H}), 2.14(\mathrm{~s}, 3 \mathrm{H}), 2.12(\mathrm{~s}$, $3 \mathrm{H}) ;{ }^{13} \mathrm{C} \mathrm{NMR}\left(75 \mathrm{MHz}, \mathrm{CDCl}_{3}\right) \delta 142.2,138.6,137.6,134.7,130.5,128.1,128.0,127.1,127.0$, $126.5,126.3,27.6,19.3$.

I. C. Burkow, L. K. Sydnes, D. C. N. Ubeda, Acta Chem. Scand. 1987, B41, 235.

\section{Table 3, entry 13}




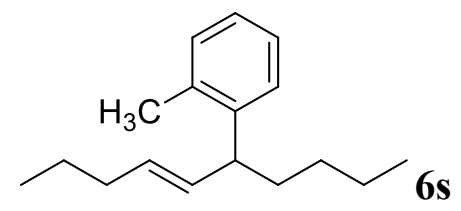

Colorless oil (0.024 g, 52\%); IR (film) 1460, $1378 \mathrm{~cm}^{-1} ;{ }^{1} \mathrm{H}$ NMR (400 MHz, $\left.\mathrm{CDCl}_{3}\right) \delta 7.20-$ 7.01 (m, 4H), 5.46 (dd, $J=15.2,7.6 \mathrm{~Hz}, 1 \mathrm{H}), 5.37$ (dt, $J=15.2,6.4 \mathrm{~Hz}, 1 \mathrm{H}), 3.43-3.37$ (m, $1 \mathrm{H}$ ), $2.31(\mathrm{~s}, 3 \mathrm{H}), 2.00-1.90(\mathrm{~m}, 2 \mathrm{H}), 1.71-1.60(\mathrm{~m}, 2 \mathrm{H}), 1.41-1.15(\mathrm{~m}, 6 \mathrm{H}), 0.95-0.80(\mathrm{~m}, 6 \mathrm{H}) ;{ }^{13} \mathrm{C}$ $\operatorname{NMR}\left(75 \mathrm{MHz}, \mathrm{CDCl}_{3}\right) \delta 143.8,135.8,133.9,130.4,130.1,126.3,126.2,125.7,44.1,35.5,34.8$, $30.0,22.9,22.8,19.8,14.2,13.8$.

HRMS calcd for $\mathrm{C}_{17} \mathrm{H}_{26}\left(\mathrm{M}^{+}\right)$: 230.2035; found: 230.2032 .

\section{Scheme 3, 4s}

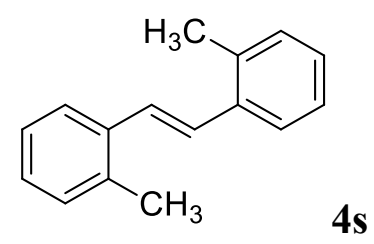

White solid (0.027 g, 65\%); m.p. $76-78{ }^{\circ} \mathrm{C}$; IR (film) 1493, 1460, $1378 \mathrm{~cm}^{-1} ;{ }^{1} \mathrm{H}$ NMR (400 $\left.\mathrm{MHz}, \mathrm{CDCl}_{3}\right) \delta 7.59(\mathrm{~d}, J=7.2 \mathrm{~Hz}, 2 \mathrm{H}), 7.25-7.16(\mathrm{~m}, 8 \mathrm{H}), 2.42(\mathrm{~s}, 6 \mathrm{H}) ;{ }^{13} \mathrm{C} \mathrm{NMR}(75 \mathrm{MHz}$, $\left.\mathrm{CDCl}_{3}\right) \delta 136.9,136.0,130.5,128.1,127.7,126.3,125.7,20.1$.

D-H. Lee, A. Taher, S. Hossain, and M-J. Jin, Org. Lett., 2011, 13, 5540.

\section{Scheme 5, 18a}

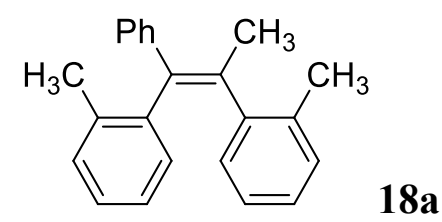

Pale yellow oil (0.040 g, 67\%); IR (film) 1598, 1491, $1440 \mathrm{~cm}^{-1} ;{ }^{1} \mathrm{H}$ NMR (400 MHz, $\left.\mathrm{CDCl}_{3}\right) \delta$ 7.38-7.28 (m, 4H), 7.26-7.18 (m, 1H), 7.10-6.81 (m, 8H), 2.31 (s, 3H), $2.16(\mathrm{~s}, 3 \mathrm{H}), 2.07(\mathrm{~s}, 3 \mathrm{H})$; ${ }^{13} \mathrm{C}$ NMR $\left(75 \mathrm{MHz}, \mathrm{CDCl}_{3}\right) \delta 136.1,134.7,130.0,129.99,129.94,127.9,126.6,126.5,126.4$, 125.3, 124.9, 22.6, 20.6, 19.8.; HRMS calcd for $\mathrm{C}_{23} \mathrm{H}_{22}\left(\mathrm{M}^{+}\right)$: 298.1722; found: 298.1722. 


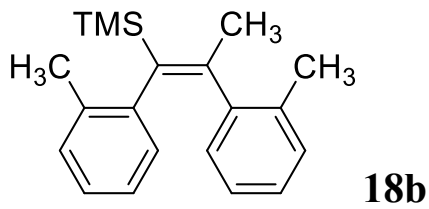

Colorless oil (0.037 g, 63\%); IR (film) 1457, $1375 \mathrm{~cm}^{-1}$; ${ }^{1} \mathrm{H}$ NMR (400 MHz, $\left.\mathrm{CDCl}_{3}\right) \delta 7.02-$

$6.60(\mathrm{~m}, 8 \mathrm{H}), 2.29(\mathrm{~s}, 3 \mathrm{H}), 2.21(\mathrm{~s}, 6 \mathrm{H}), 0.15(\mathrm{~s}, 9 \mathrm{H}) ;{ }^{13} \mathrm{C} \mathrm{NMR}\left(100 \mathrm{MHz}, \mathrm{CDCl}_{3}\right) \delta$ 148.7, $145.1,144.5,140.5,134.2,133.5,129.6,129.2,126.7,126.2,126.1,125.1,125.0,24.6,20.8$, 19.7, 0.5. 


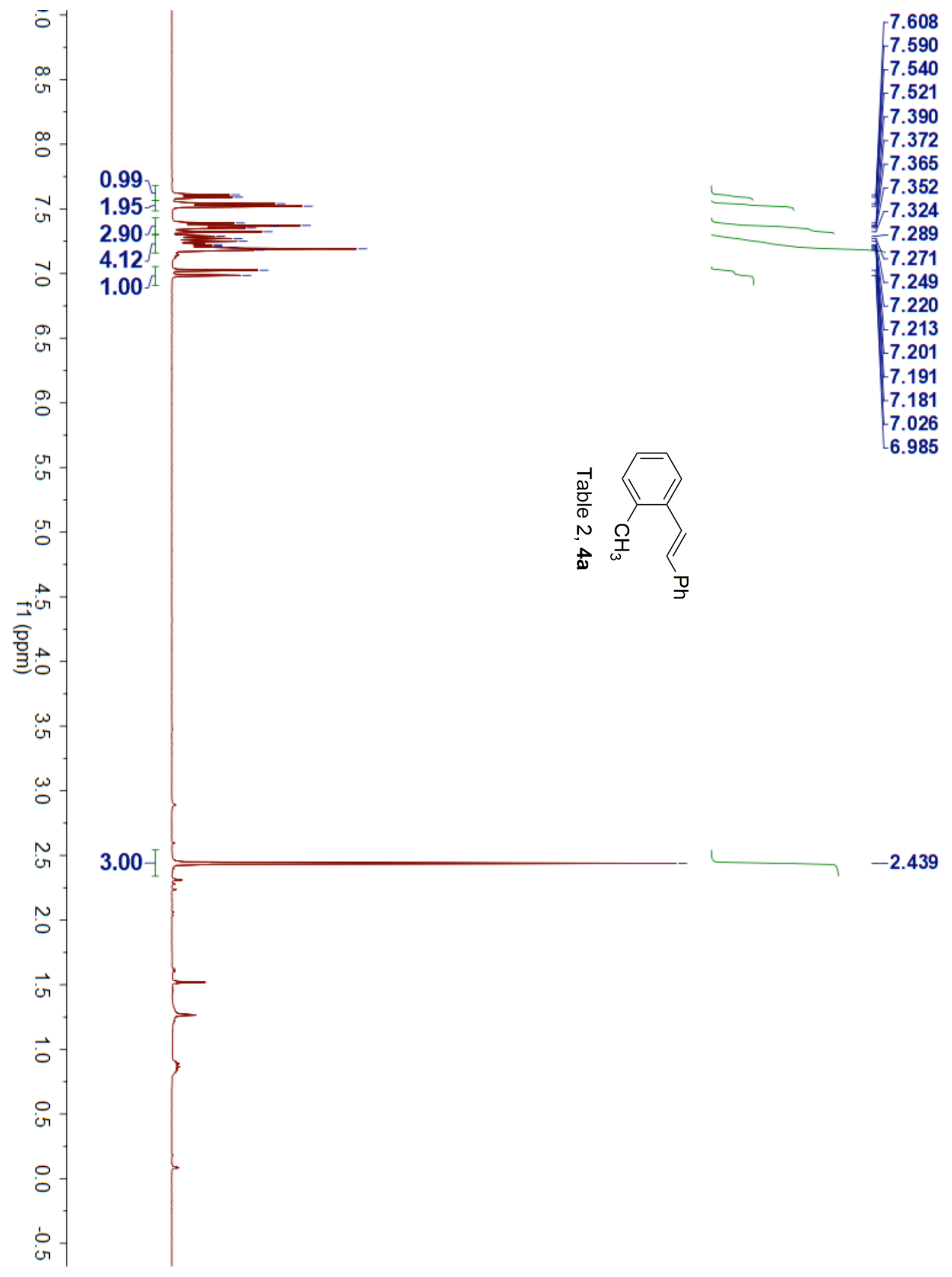




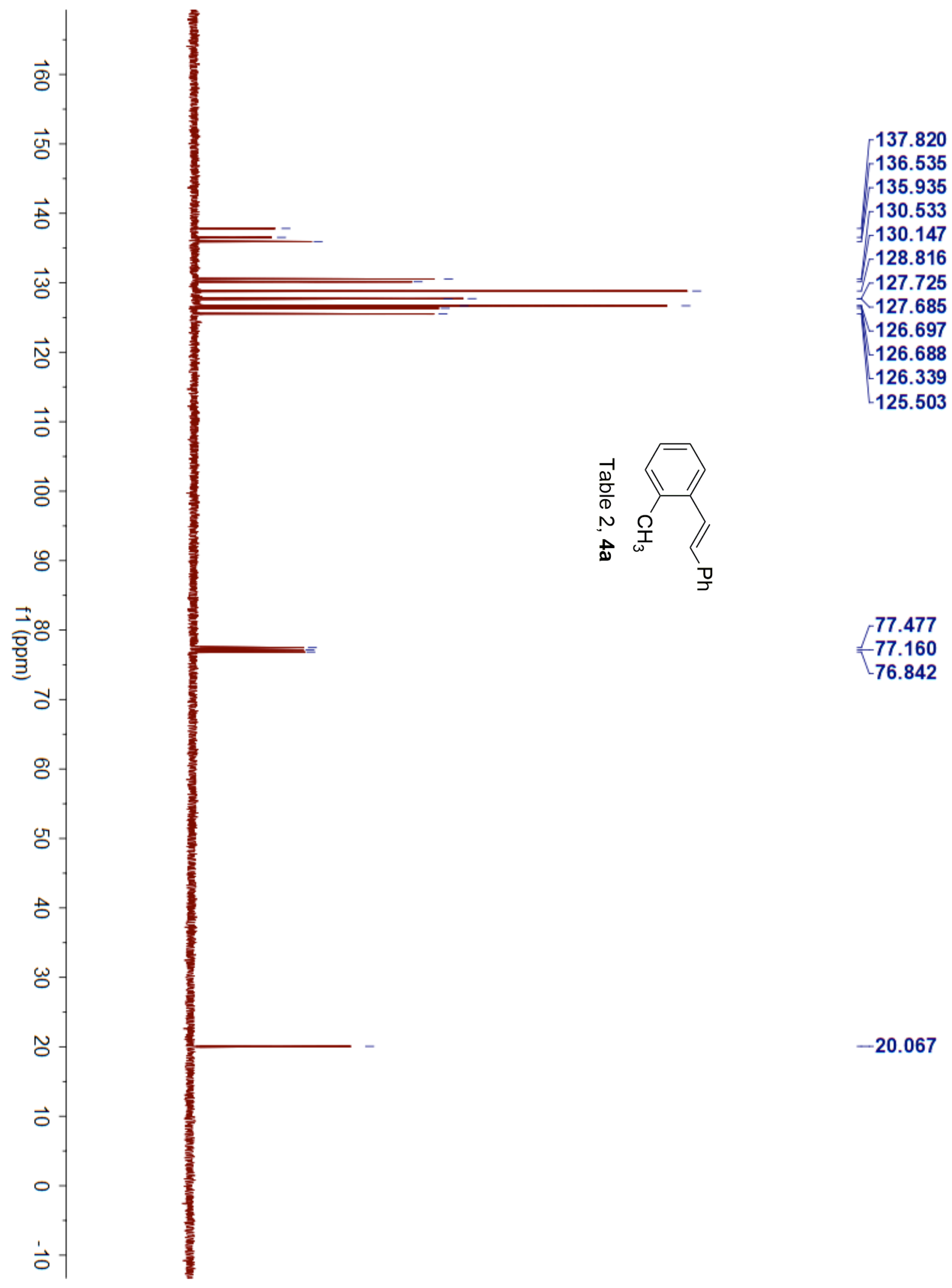




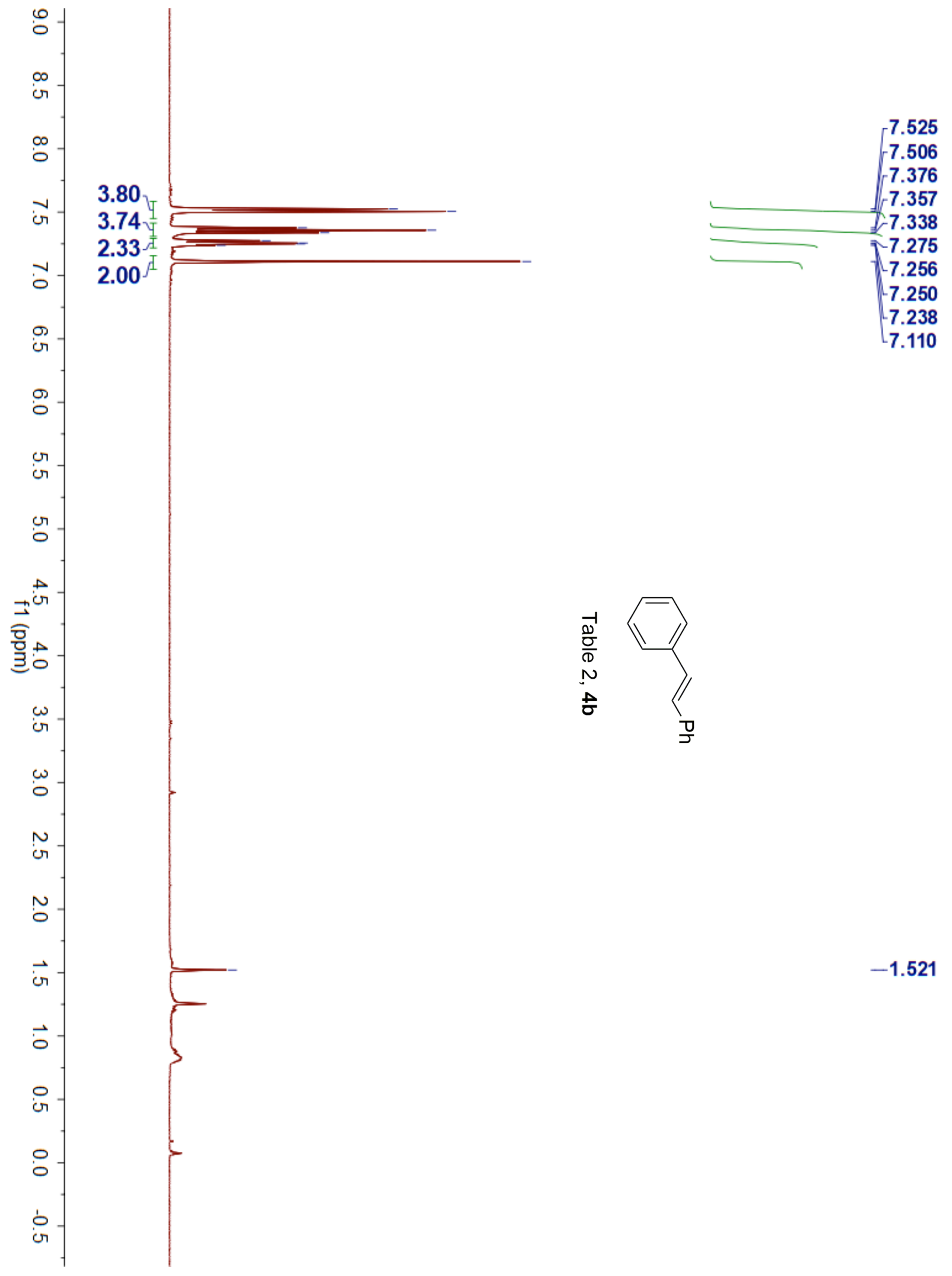




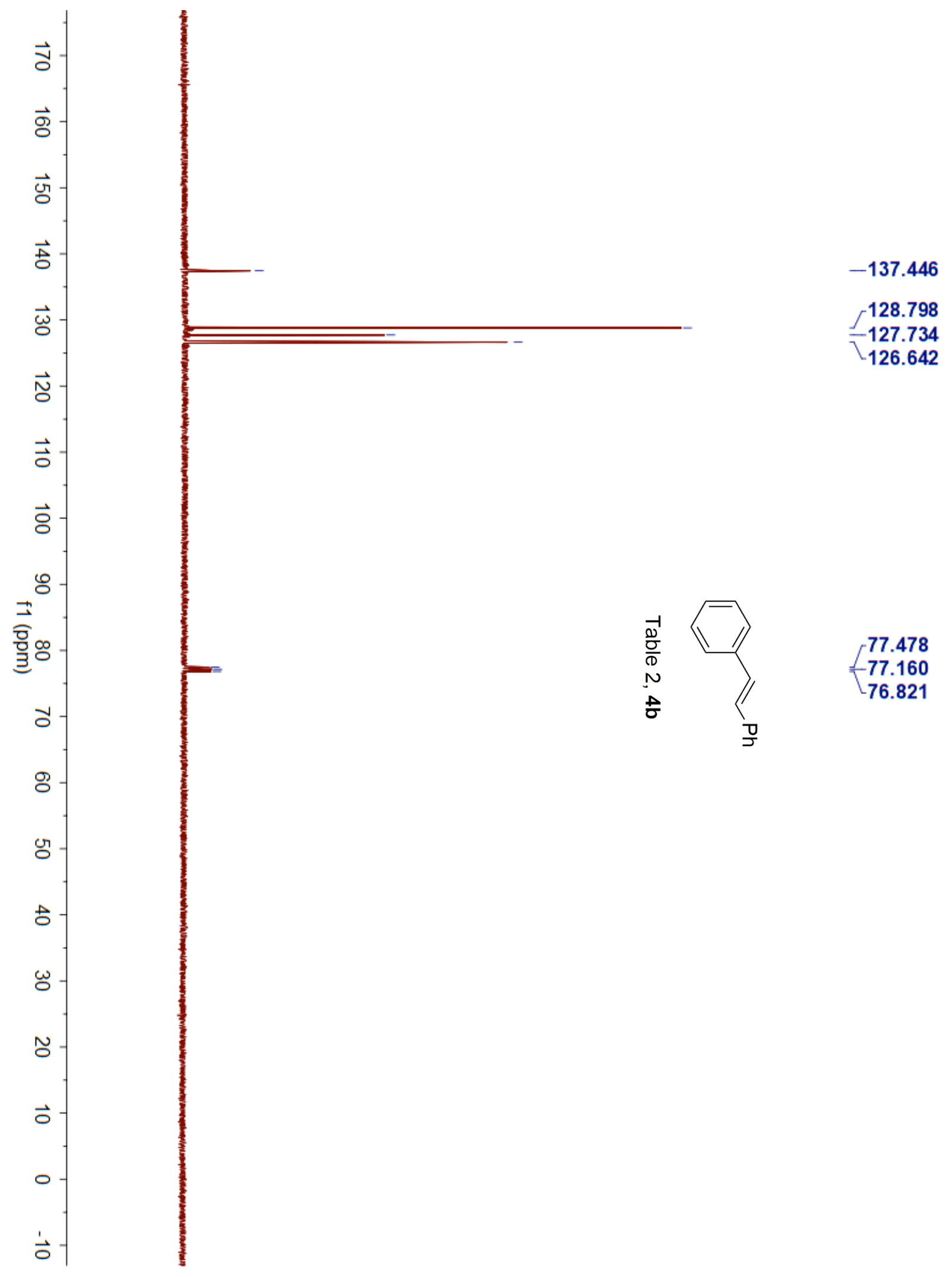




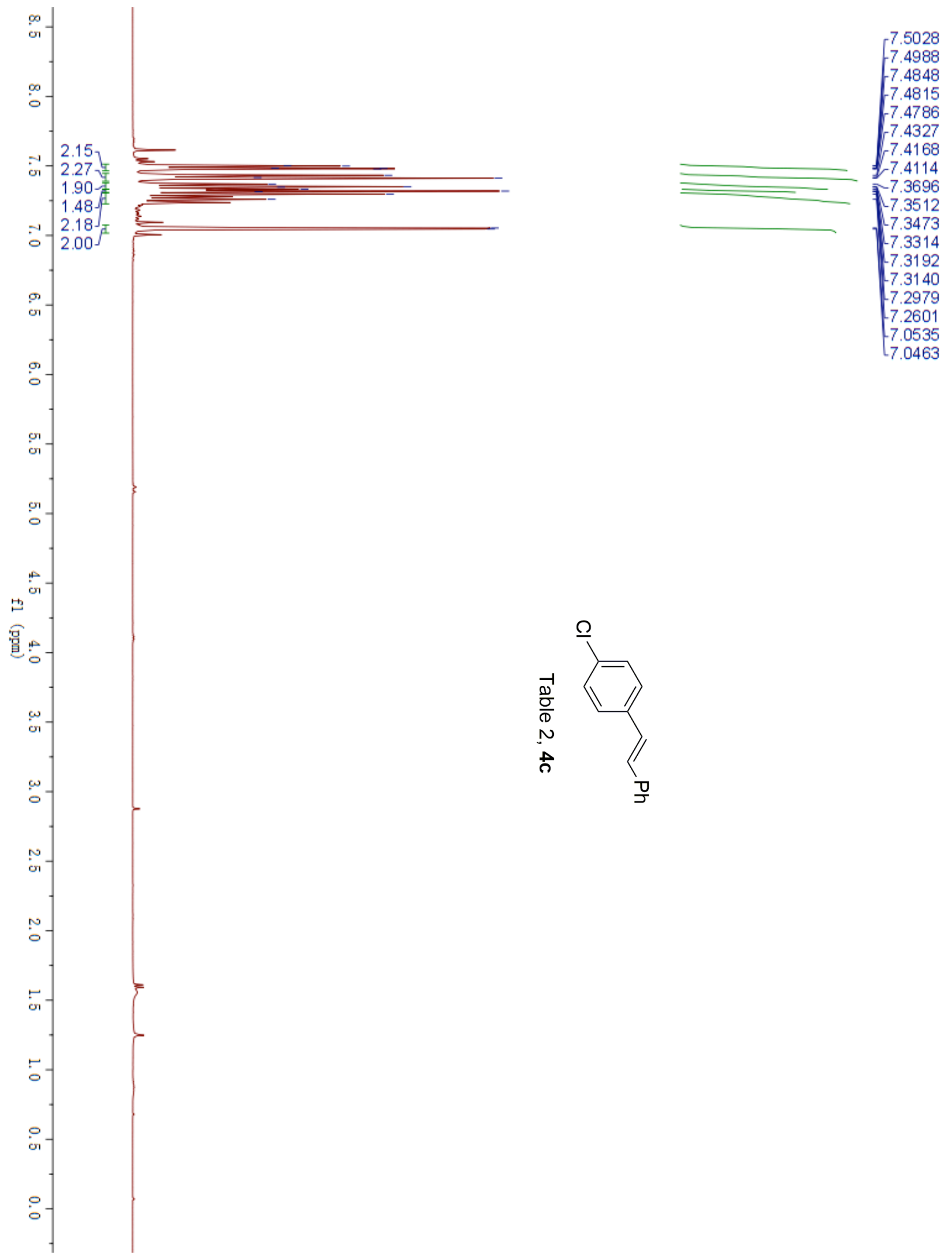




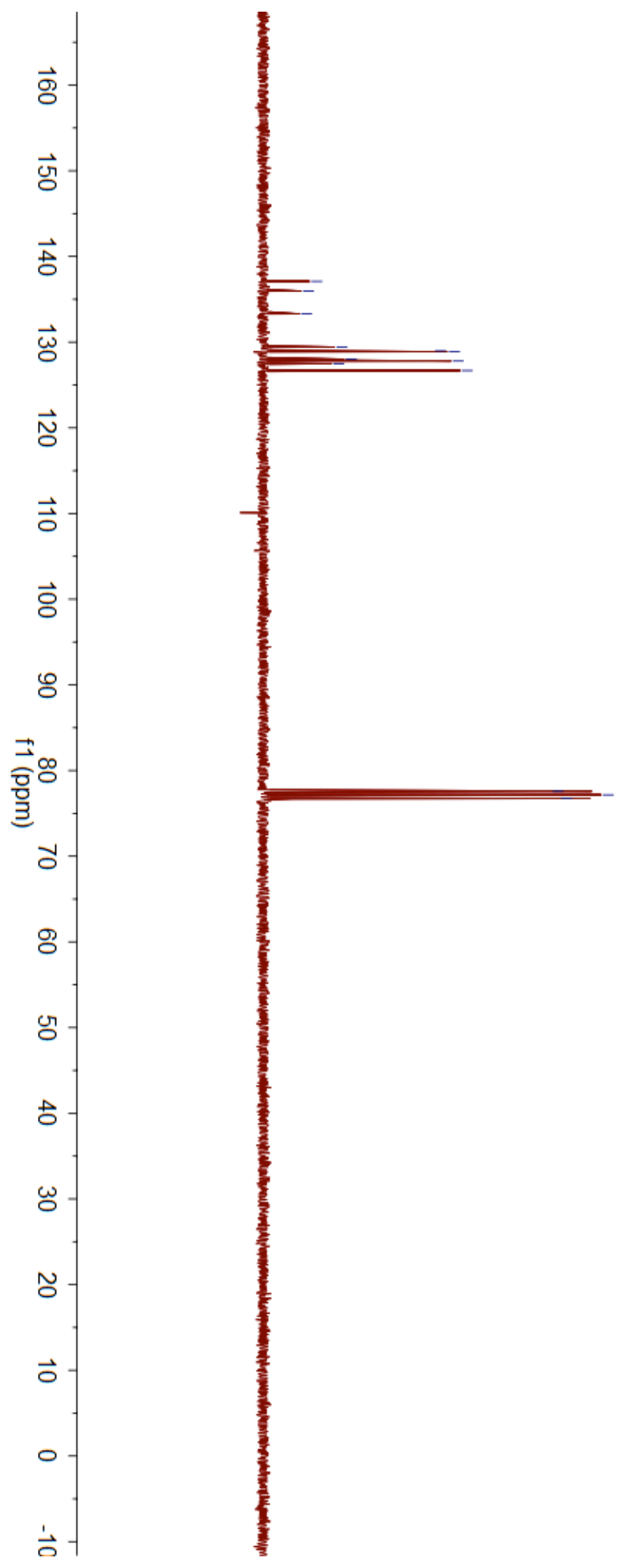

137.094 135.958 133.294 129.427 128.976 $-128.876$ 128.008 $-127.793$ 127.482

126.680

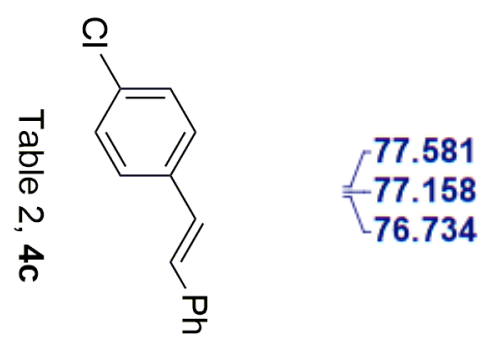




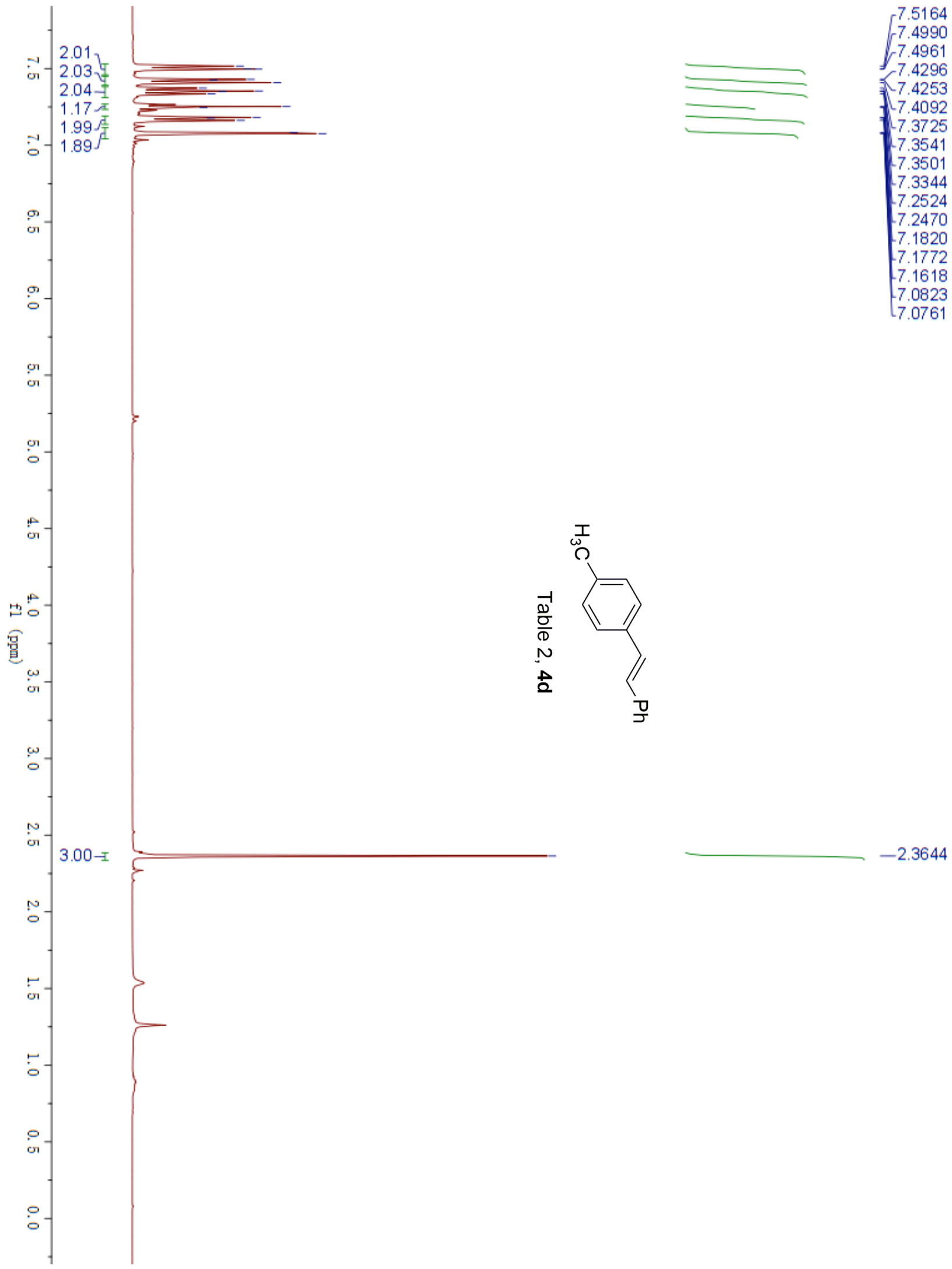




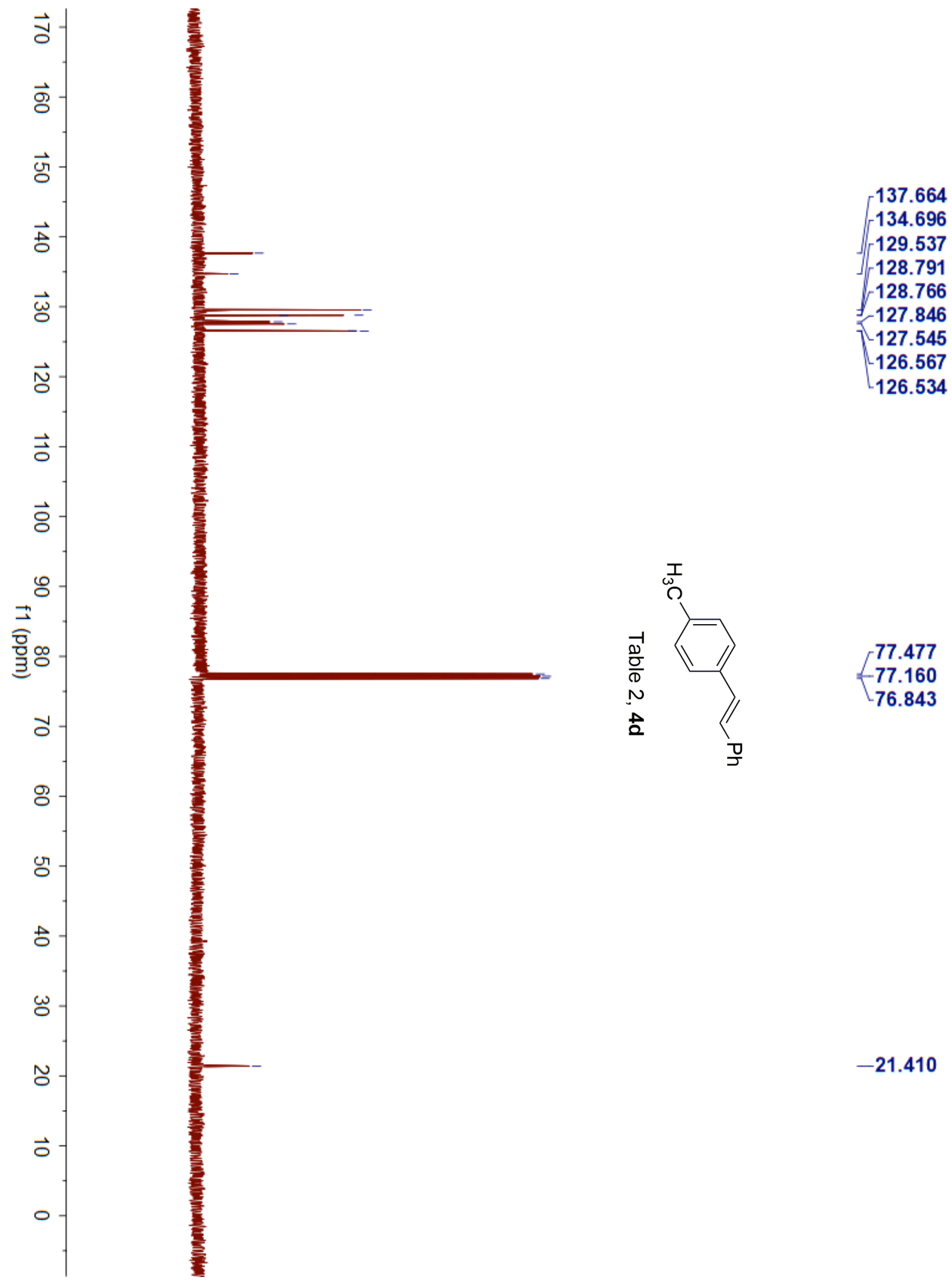




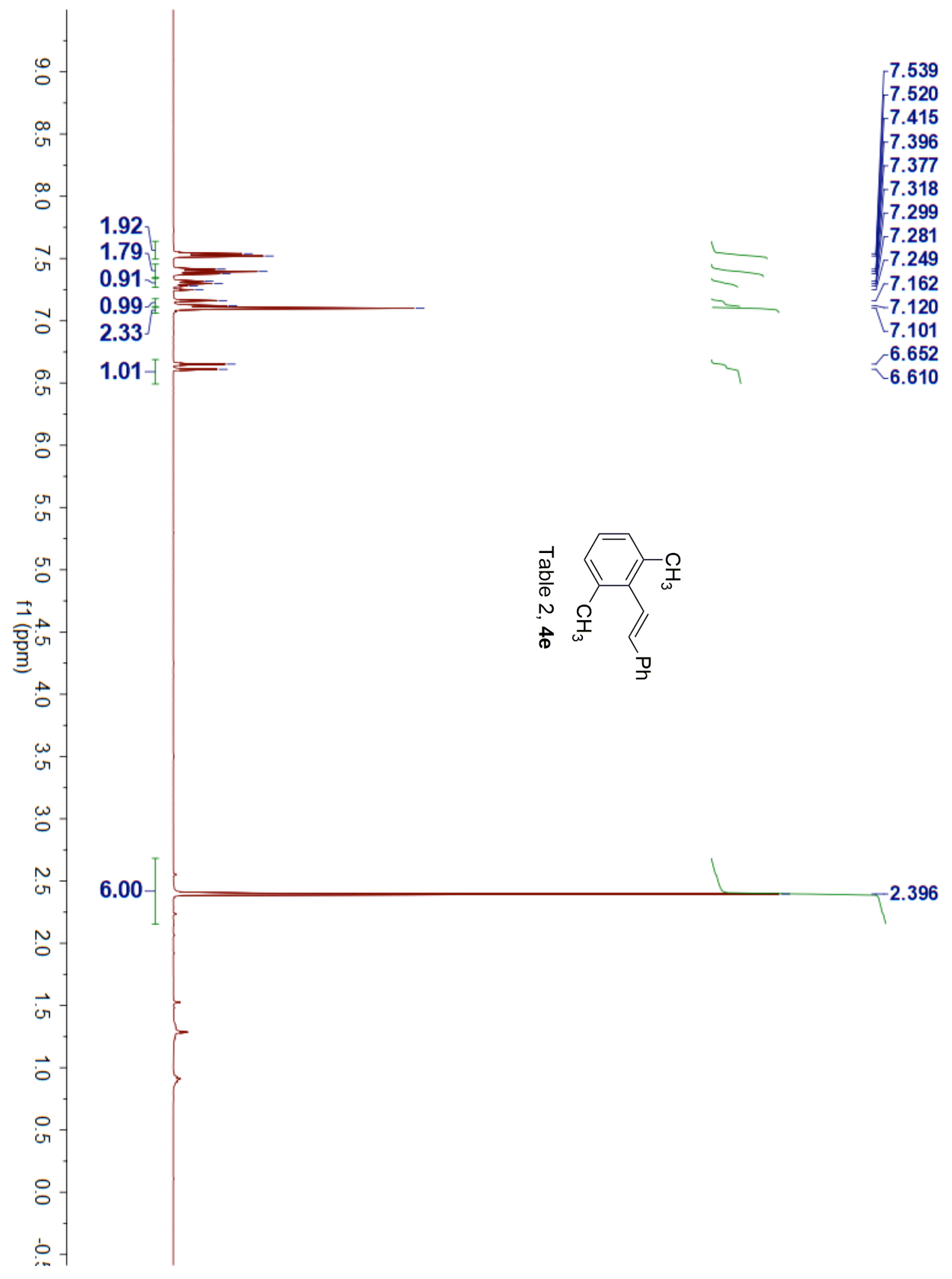




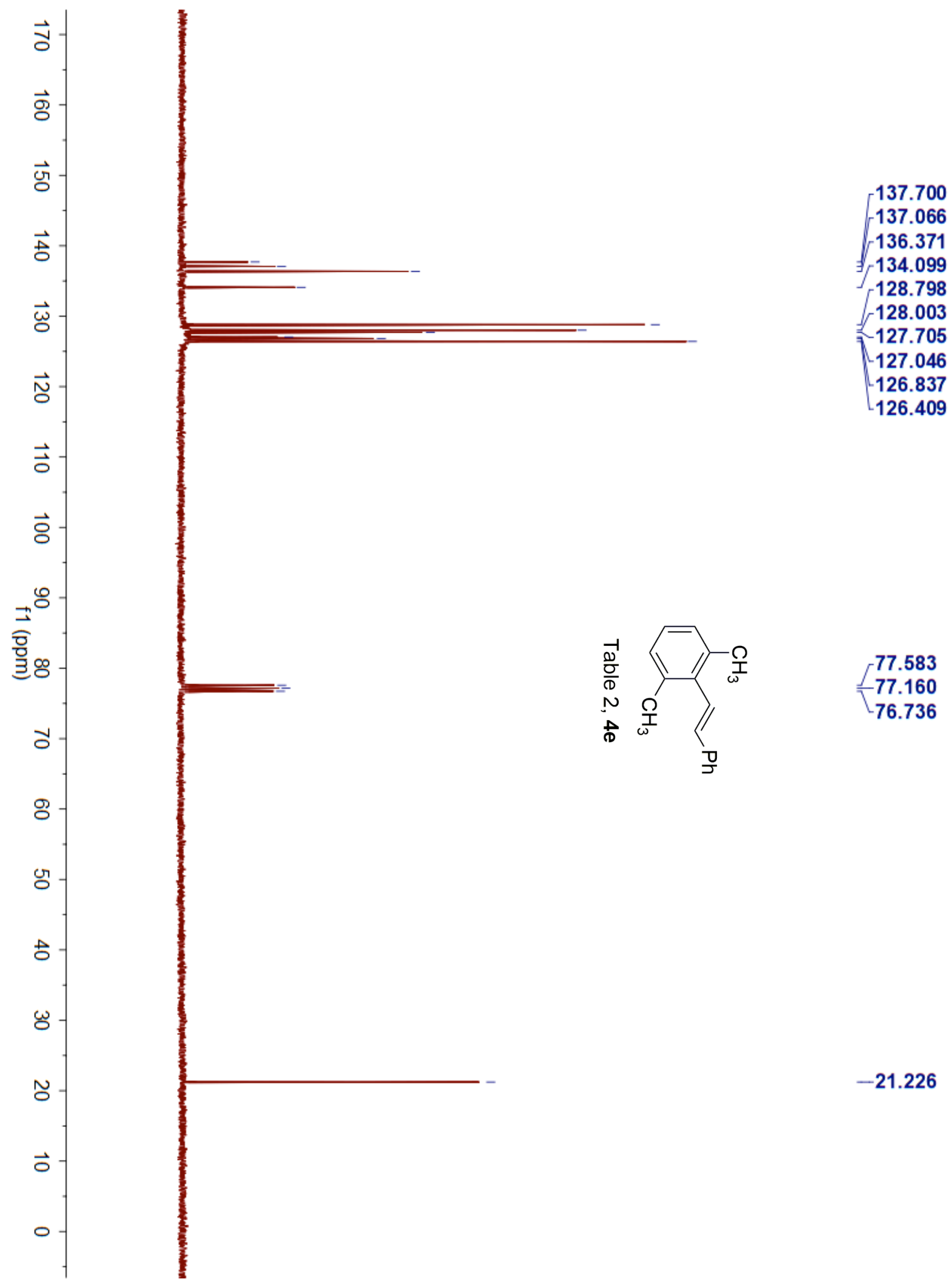




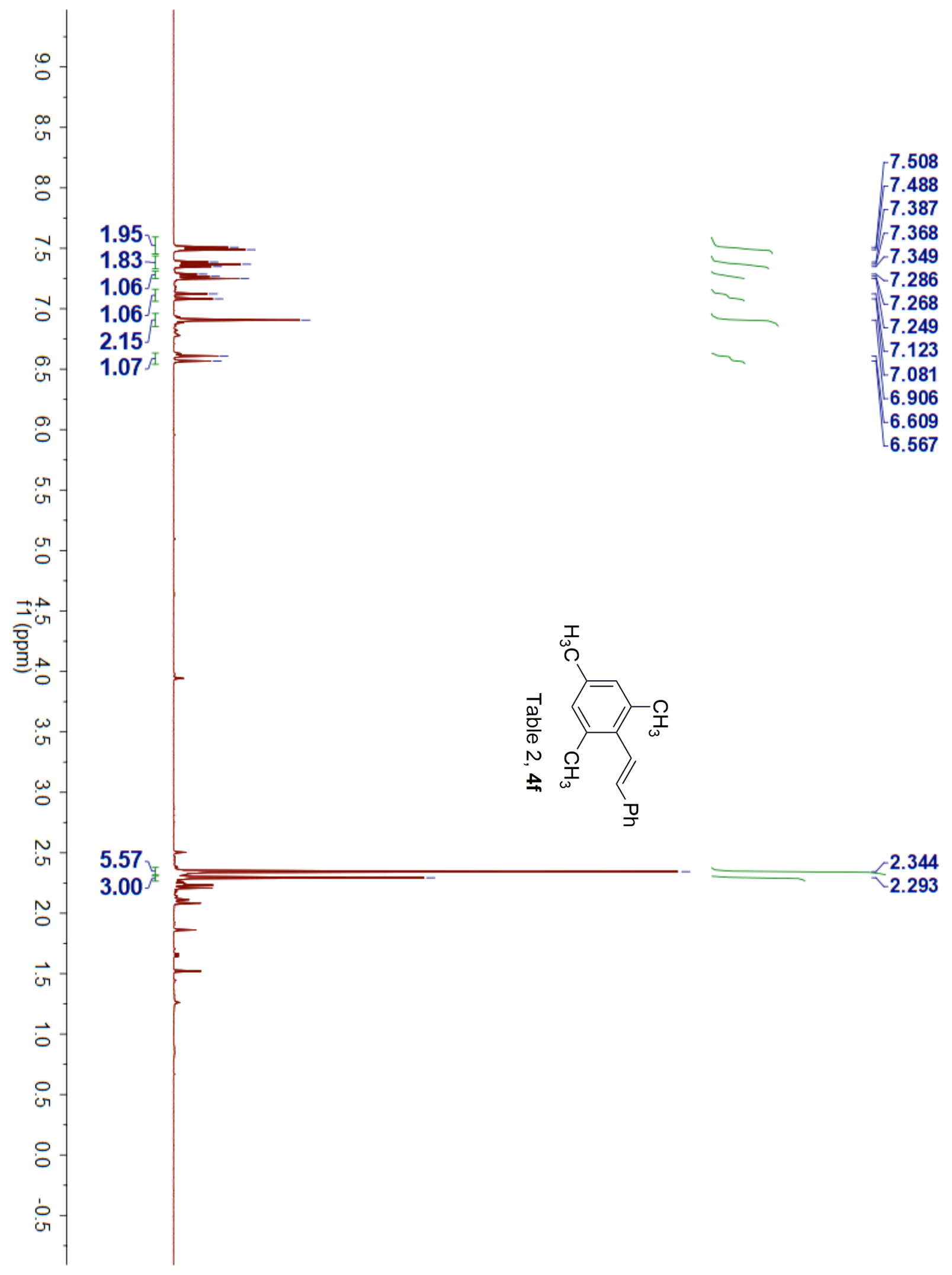




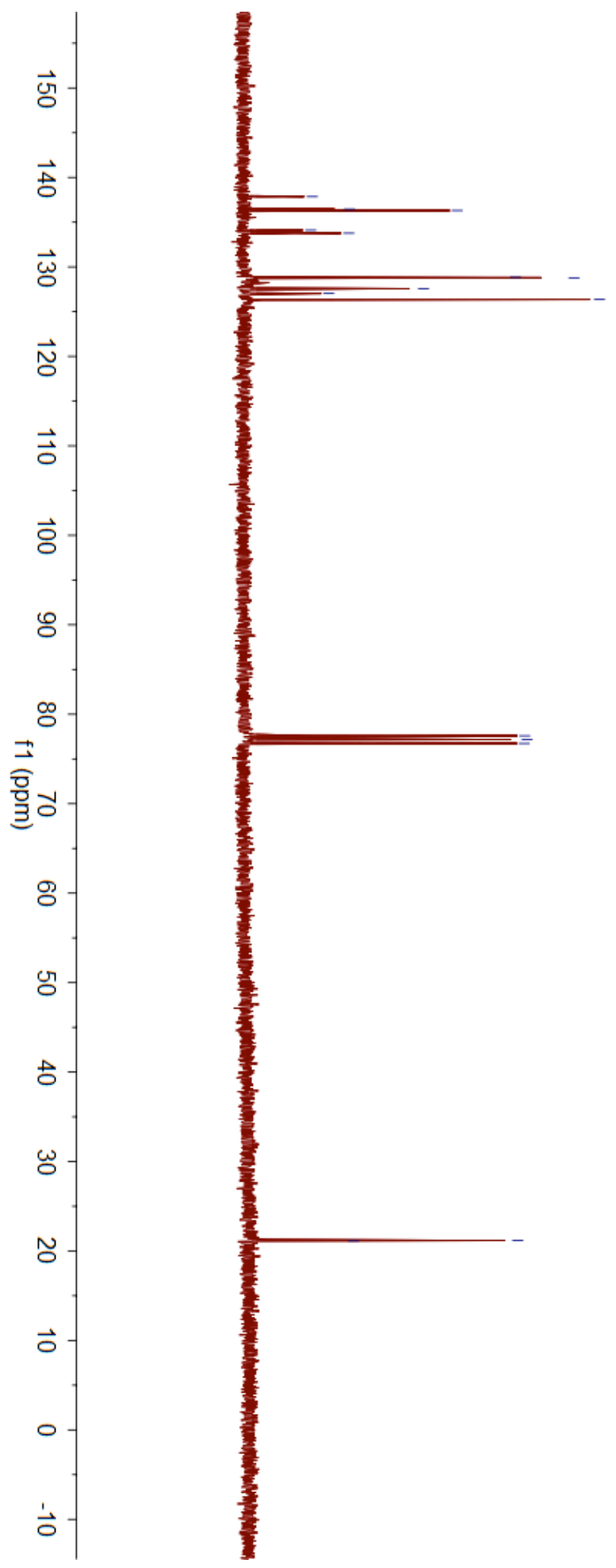

137.859

136.453

136.304

J. 134.106

$-133.773$

128.842

128.779

127.581

$-127.030$

126.358

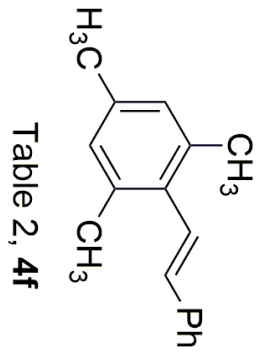

\begin{tabular}{r}
77.583 \\
\multicolumn{1}{r}{77.160} \\
76.737
\end{tabular}

21.167

21.126 


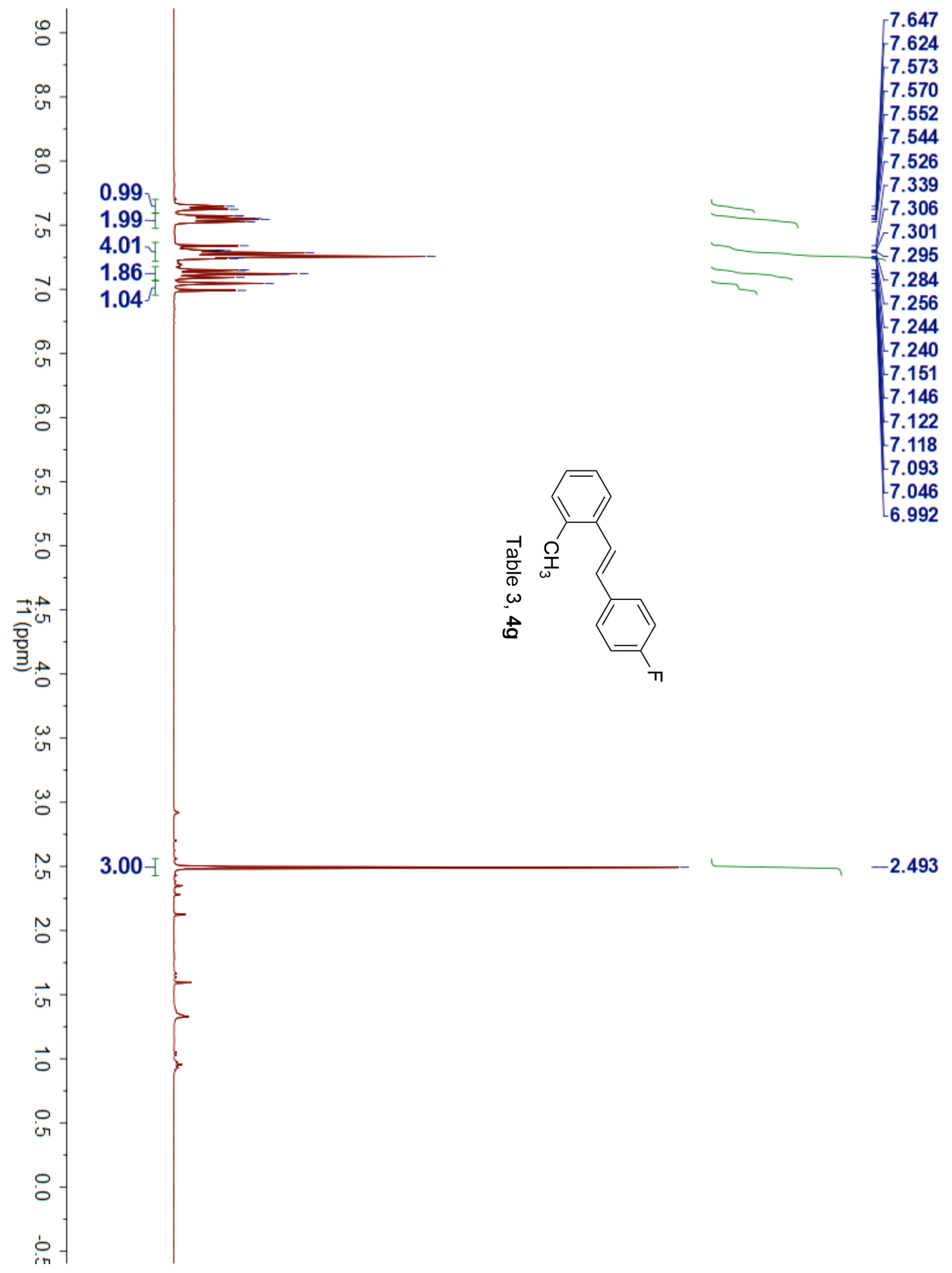




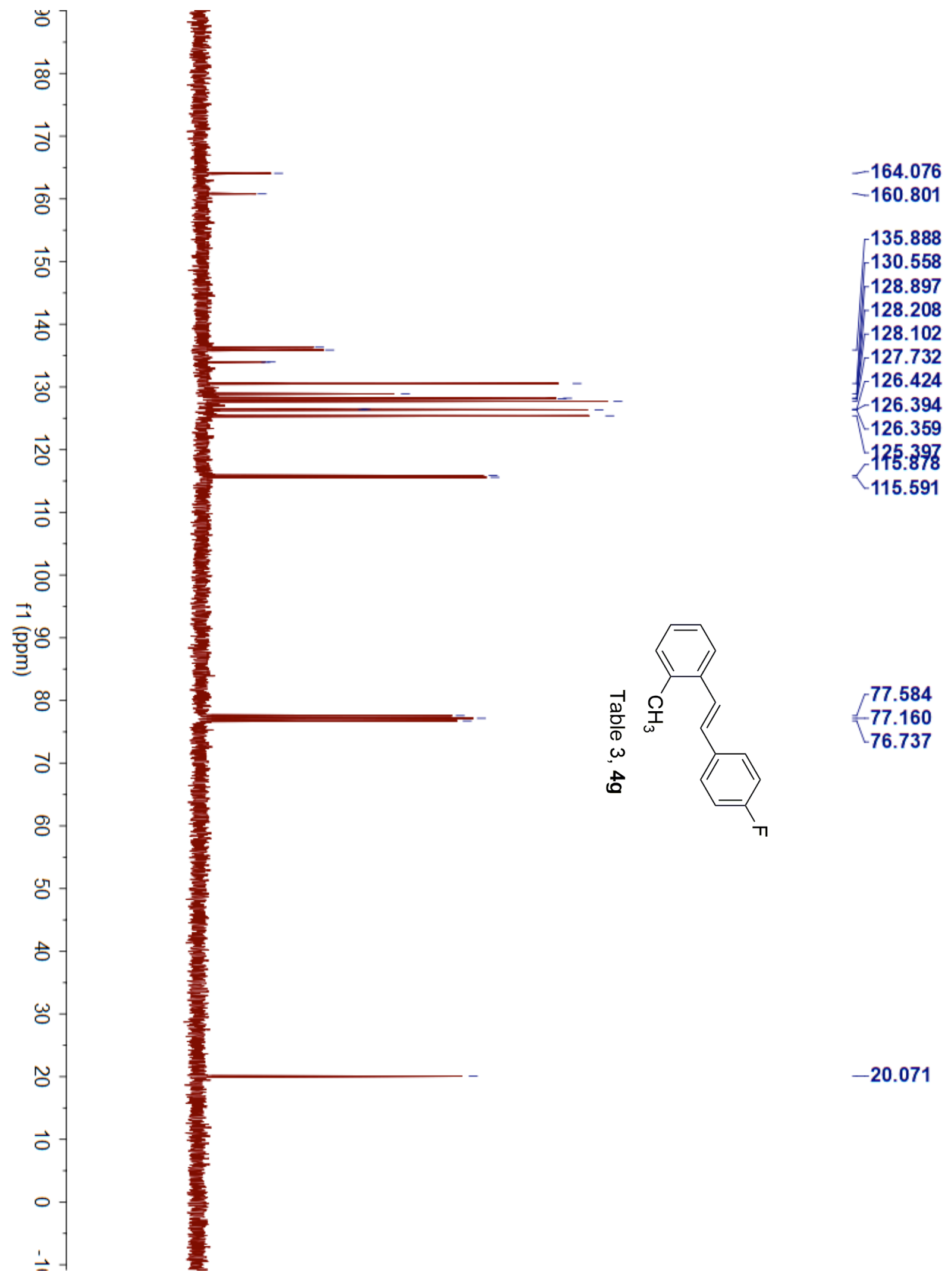




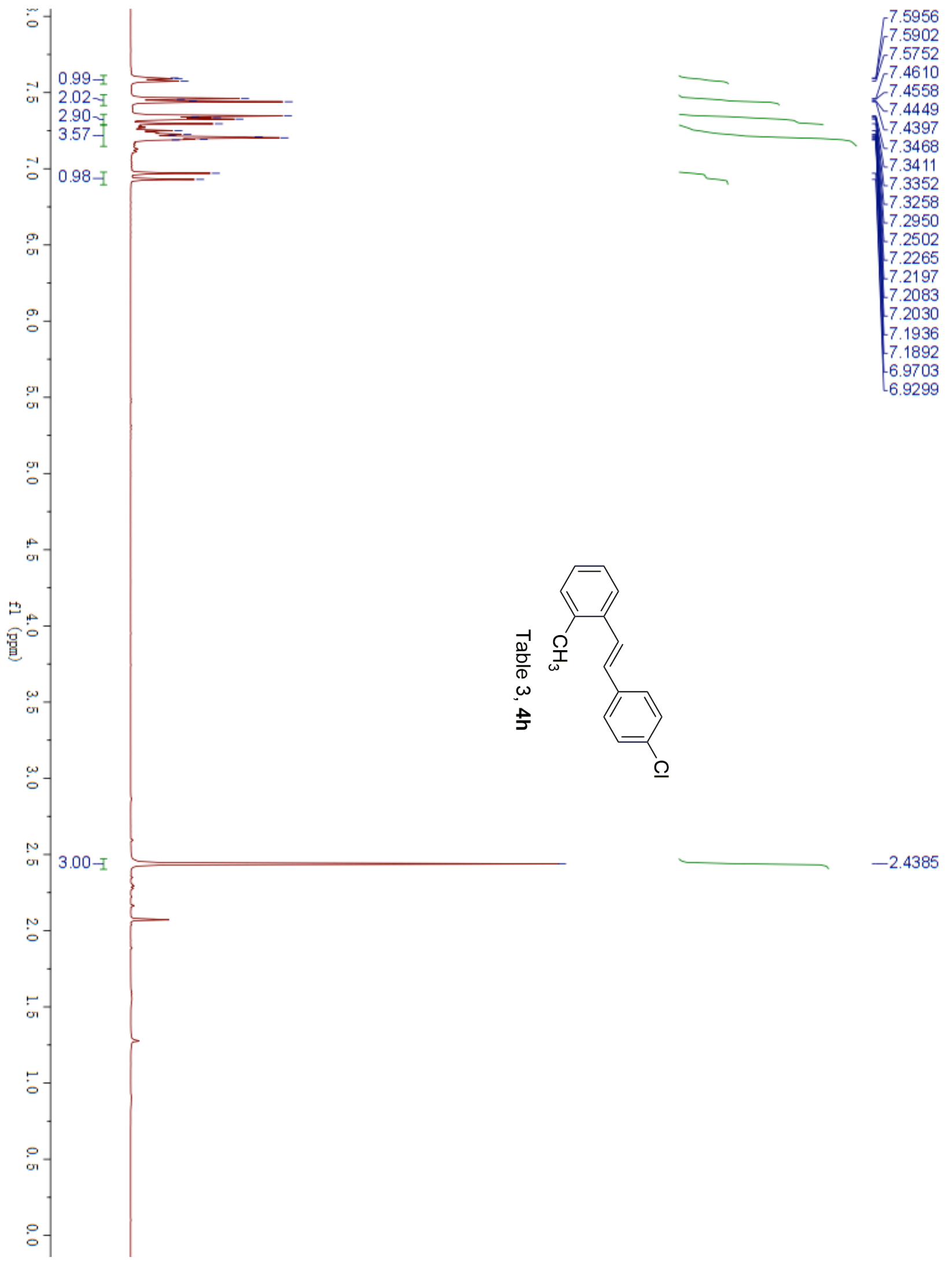




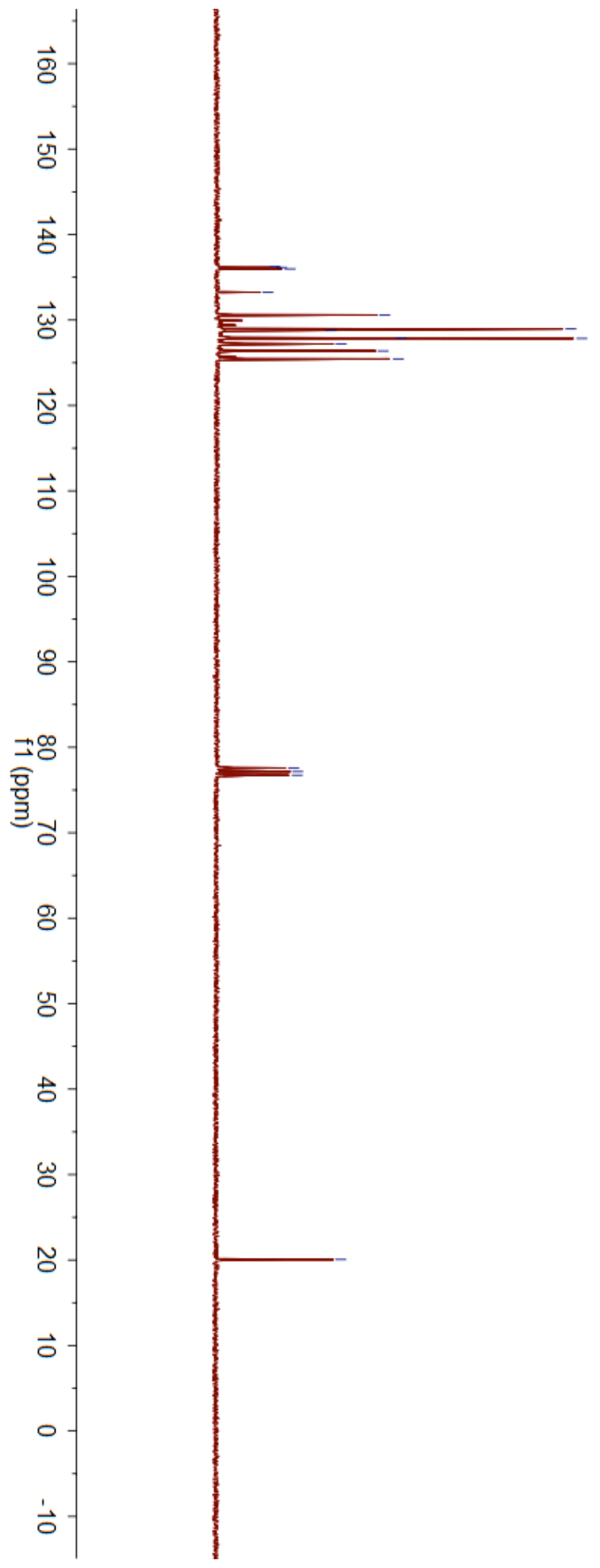

136.272

136.143

135.981

133.250

130.594

128.947

$\perp 128.769$

$-127.906$

$-127.834$

127.199

126. 375

125.447

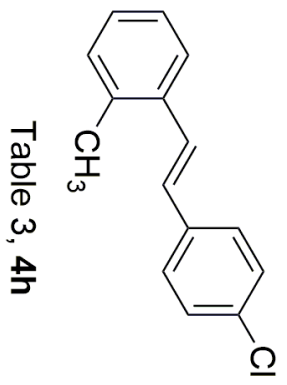

77.584

$-77.160$

76.737

$-20.062$ 


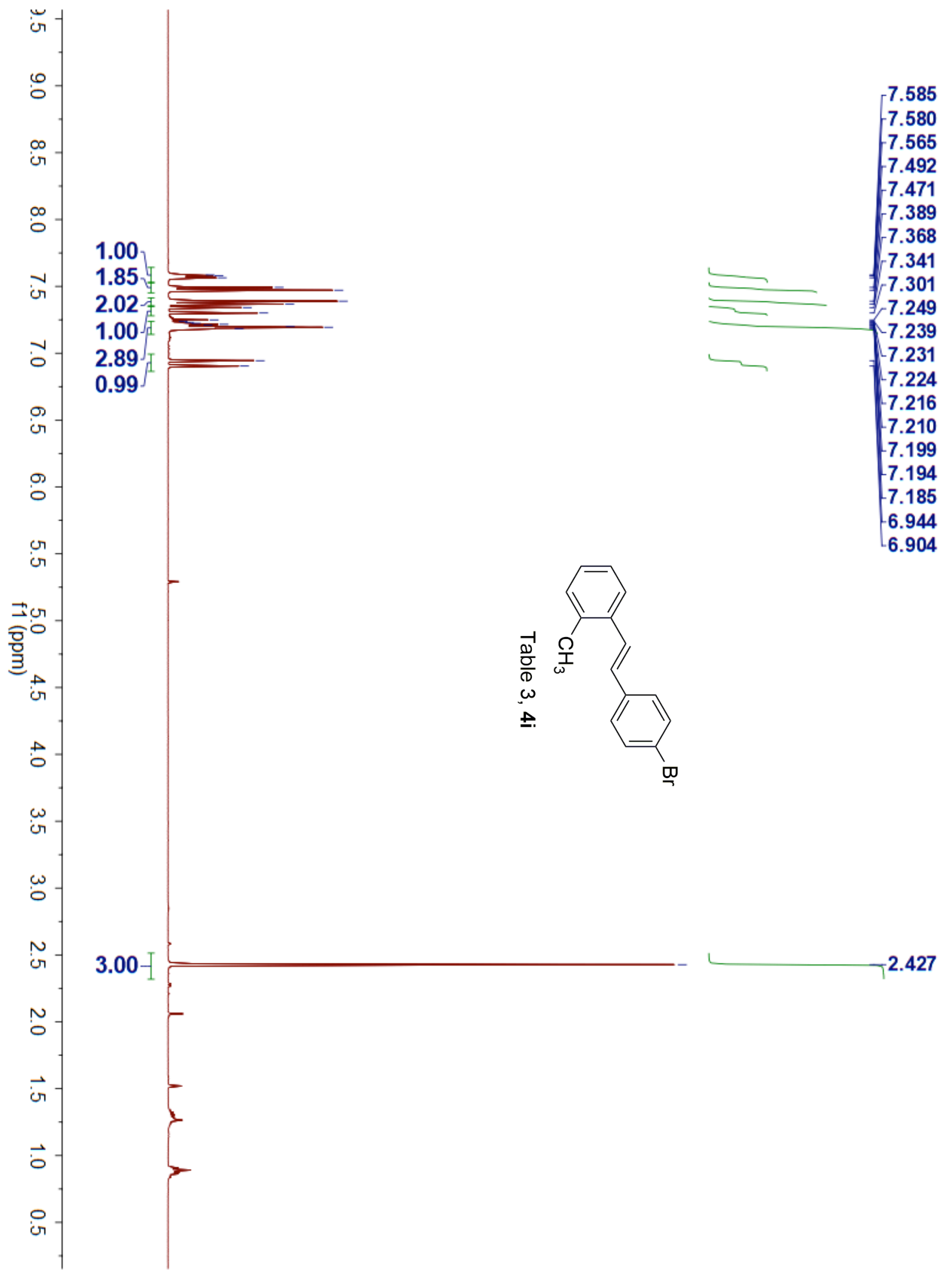




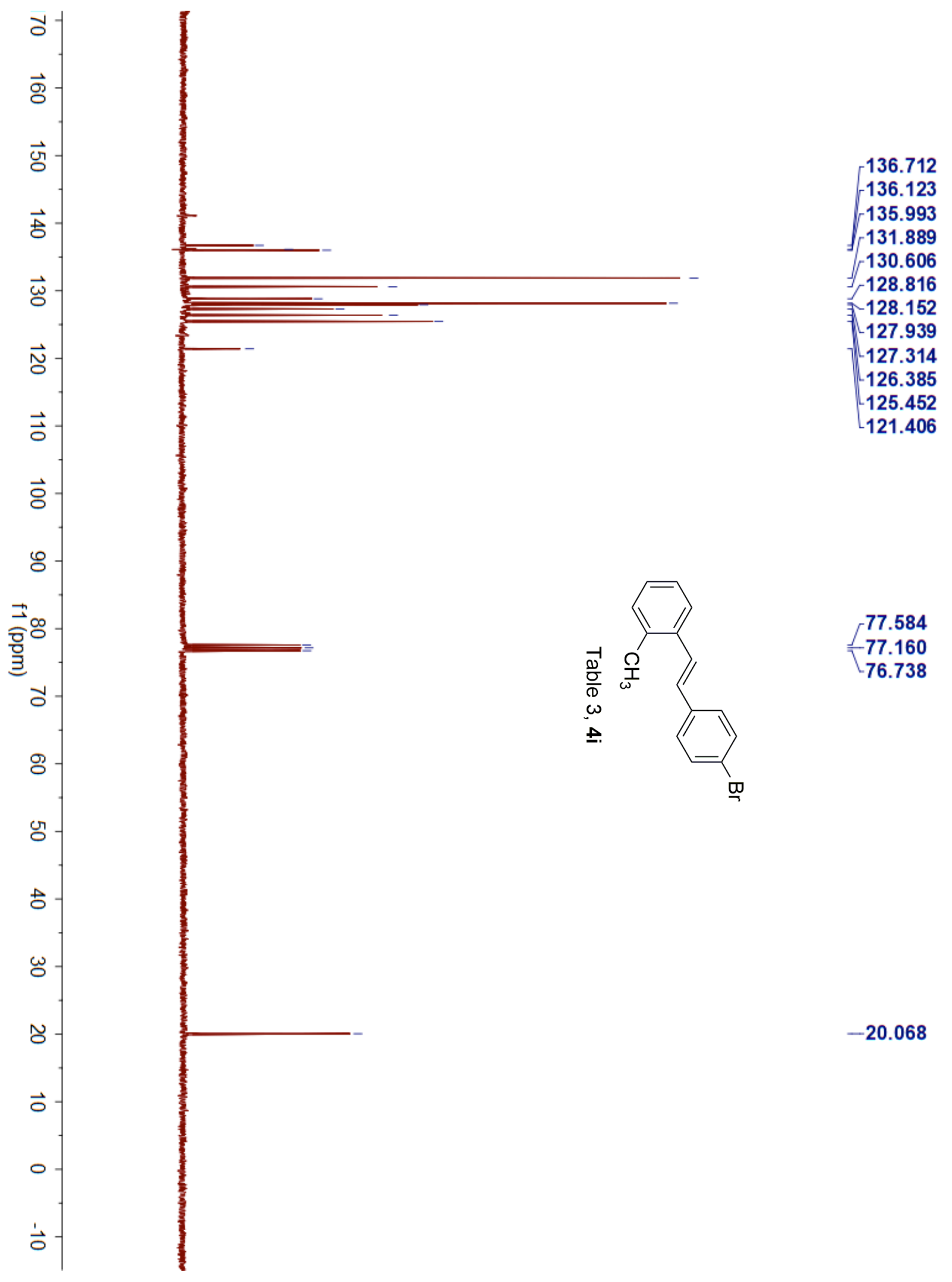




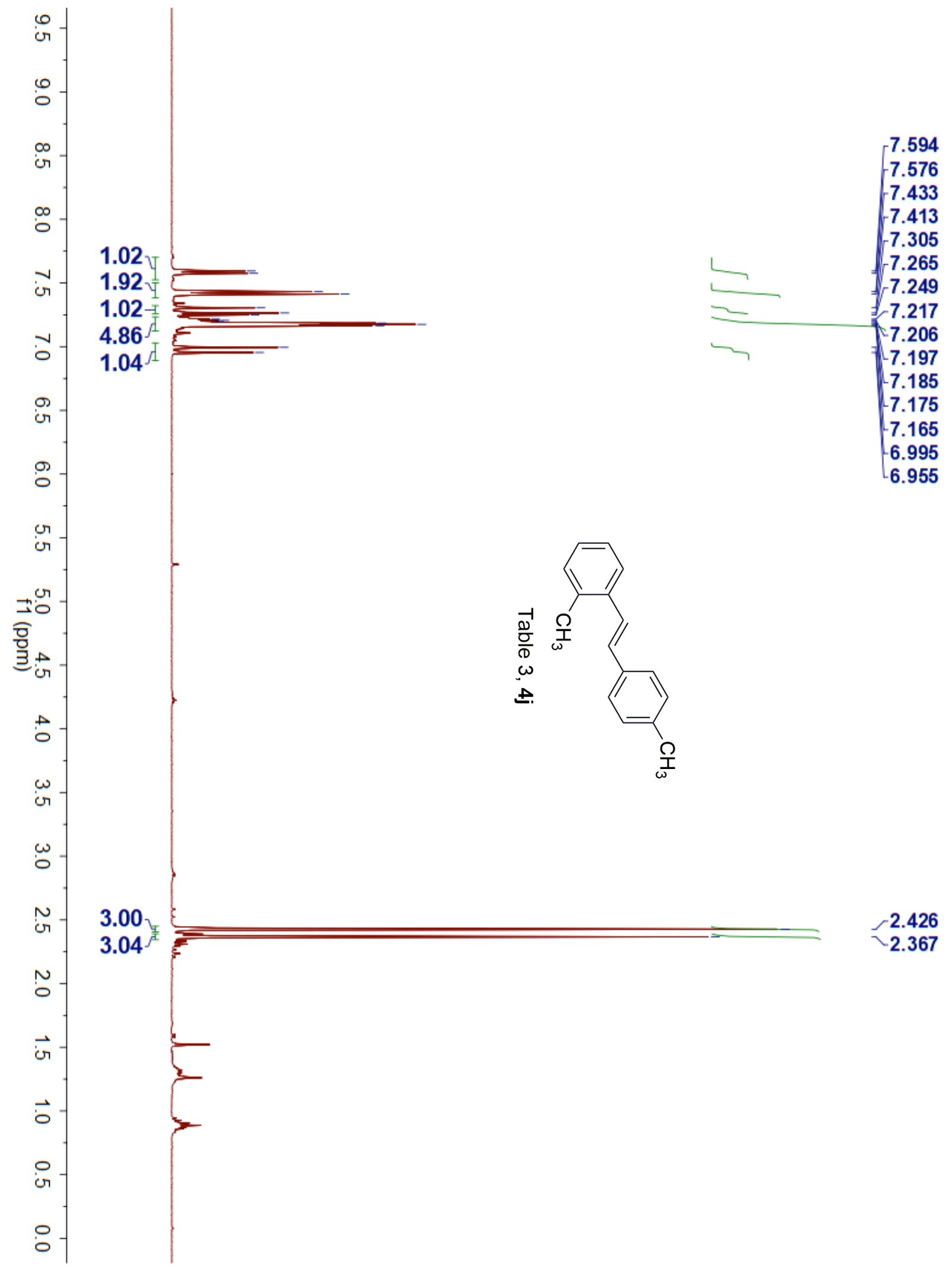




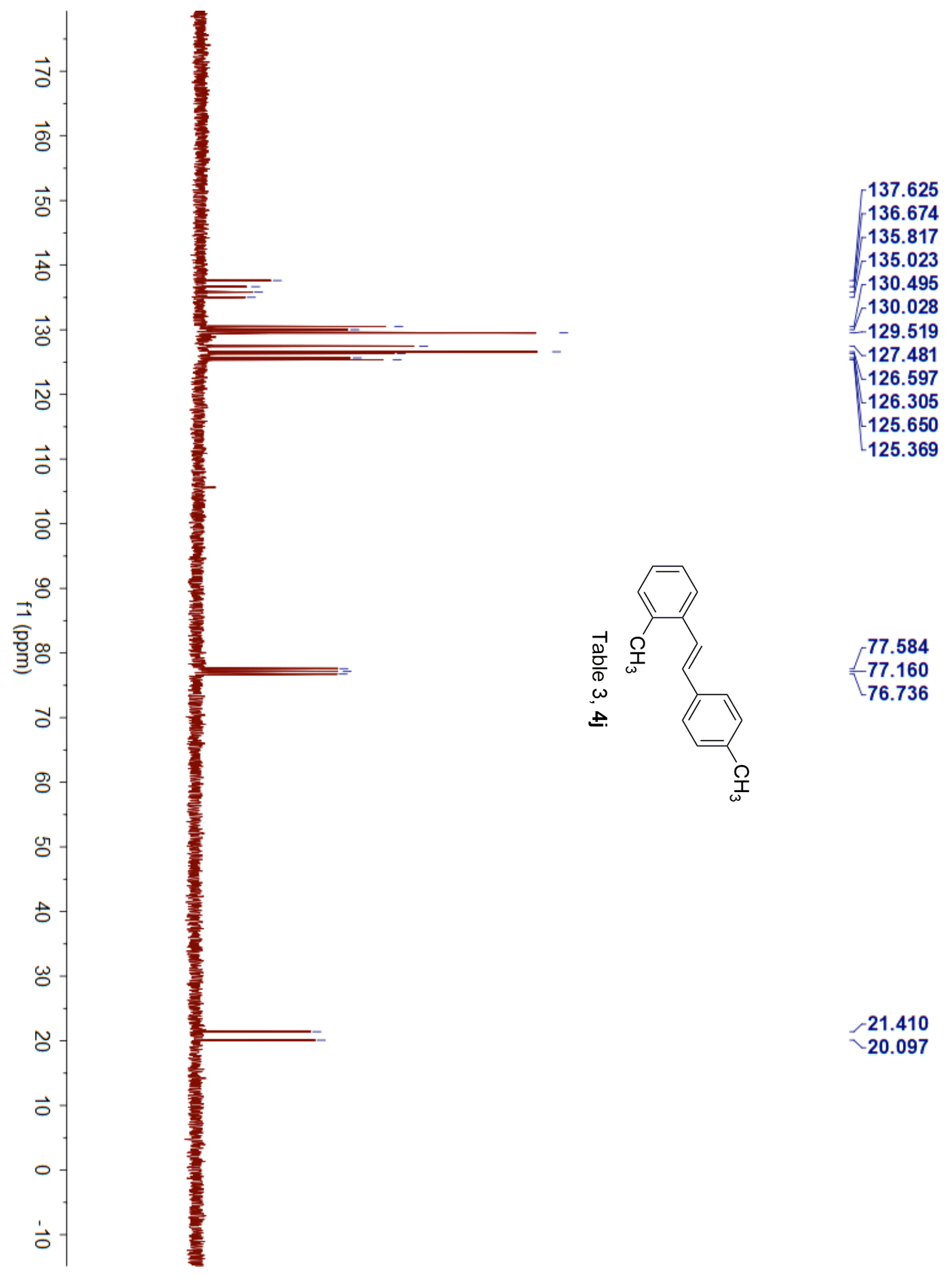




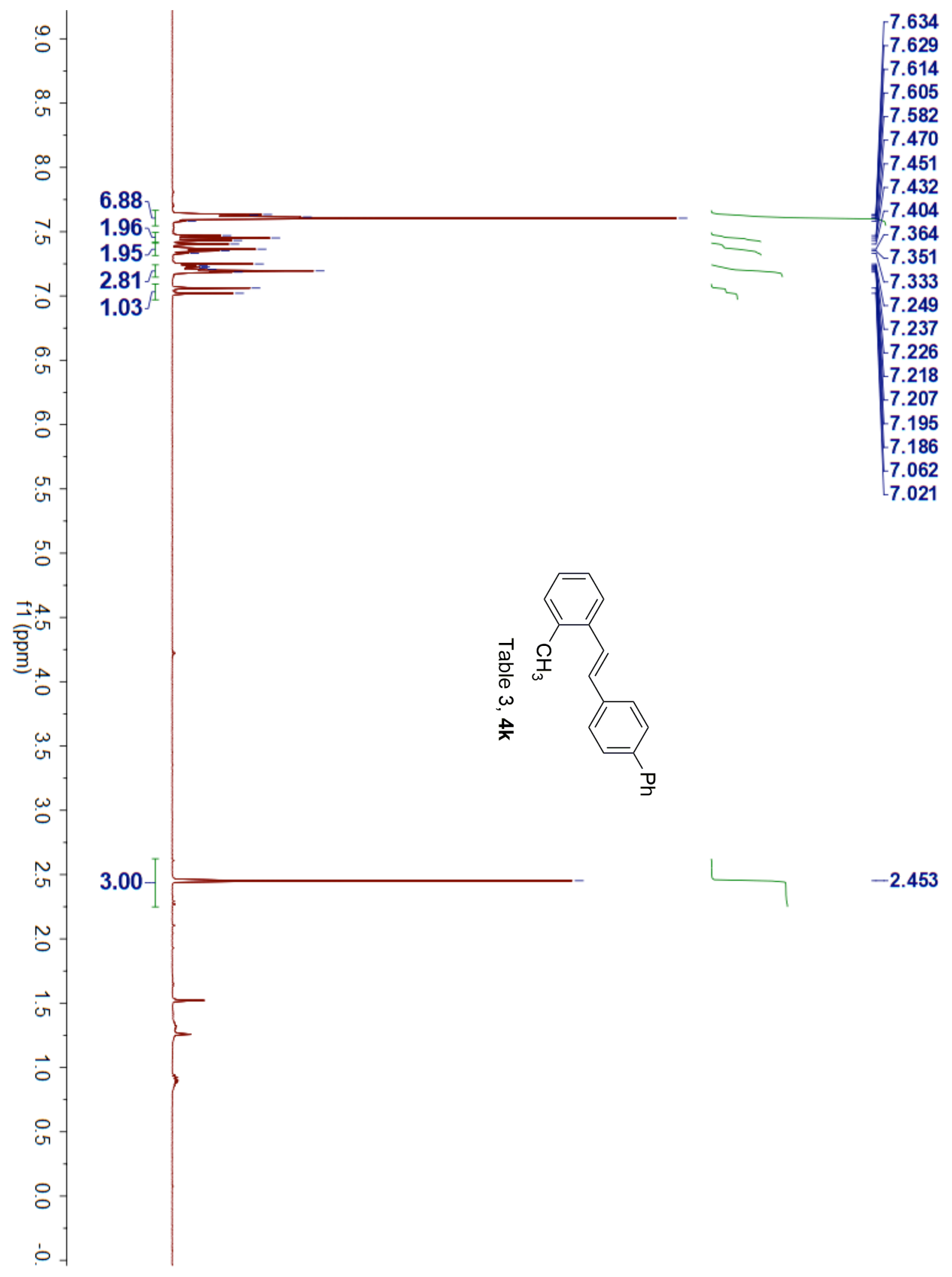




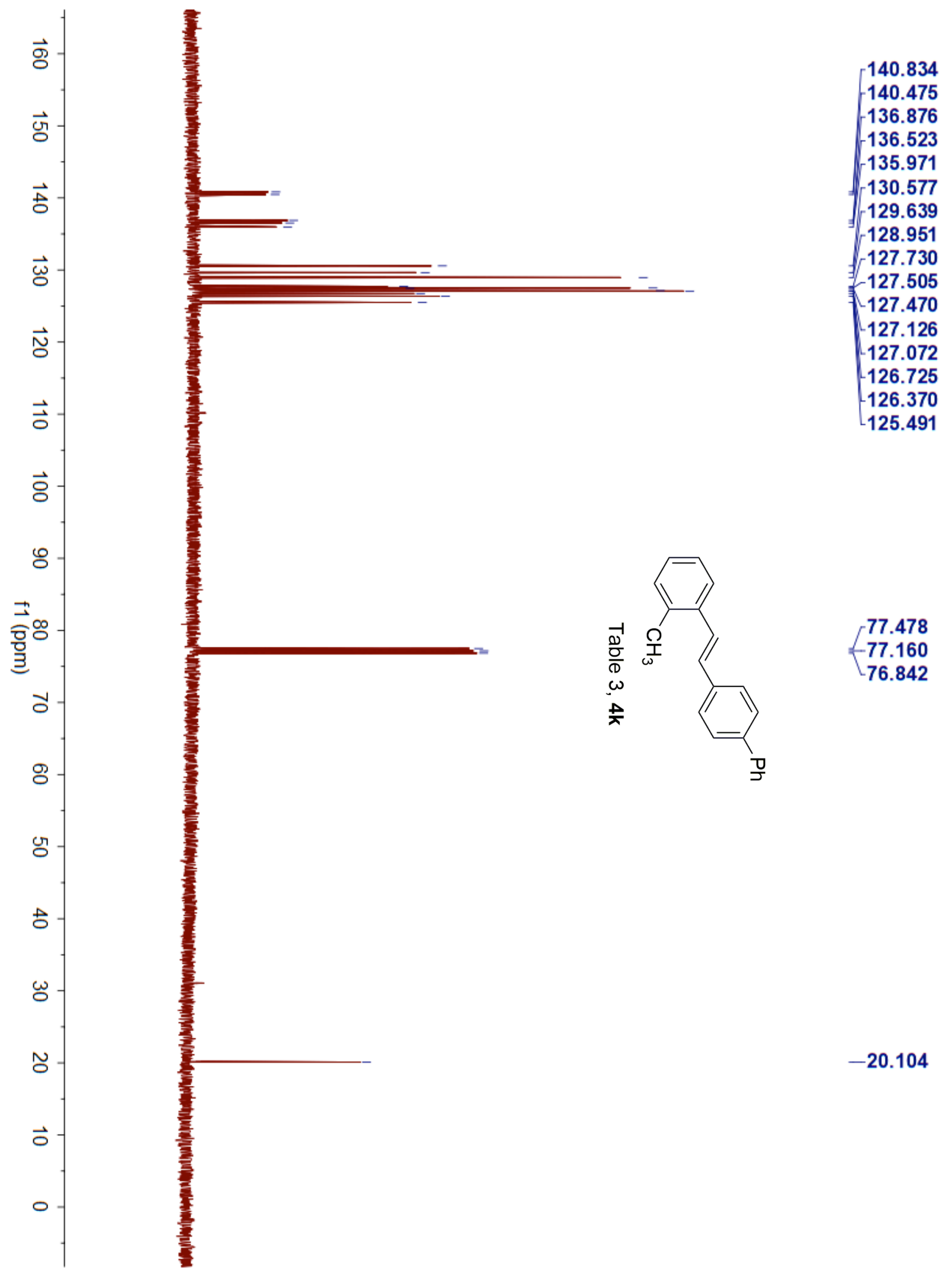




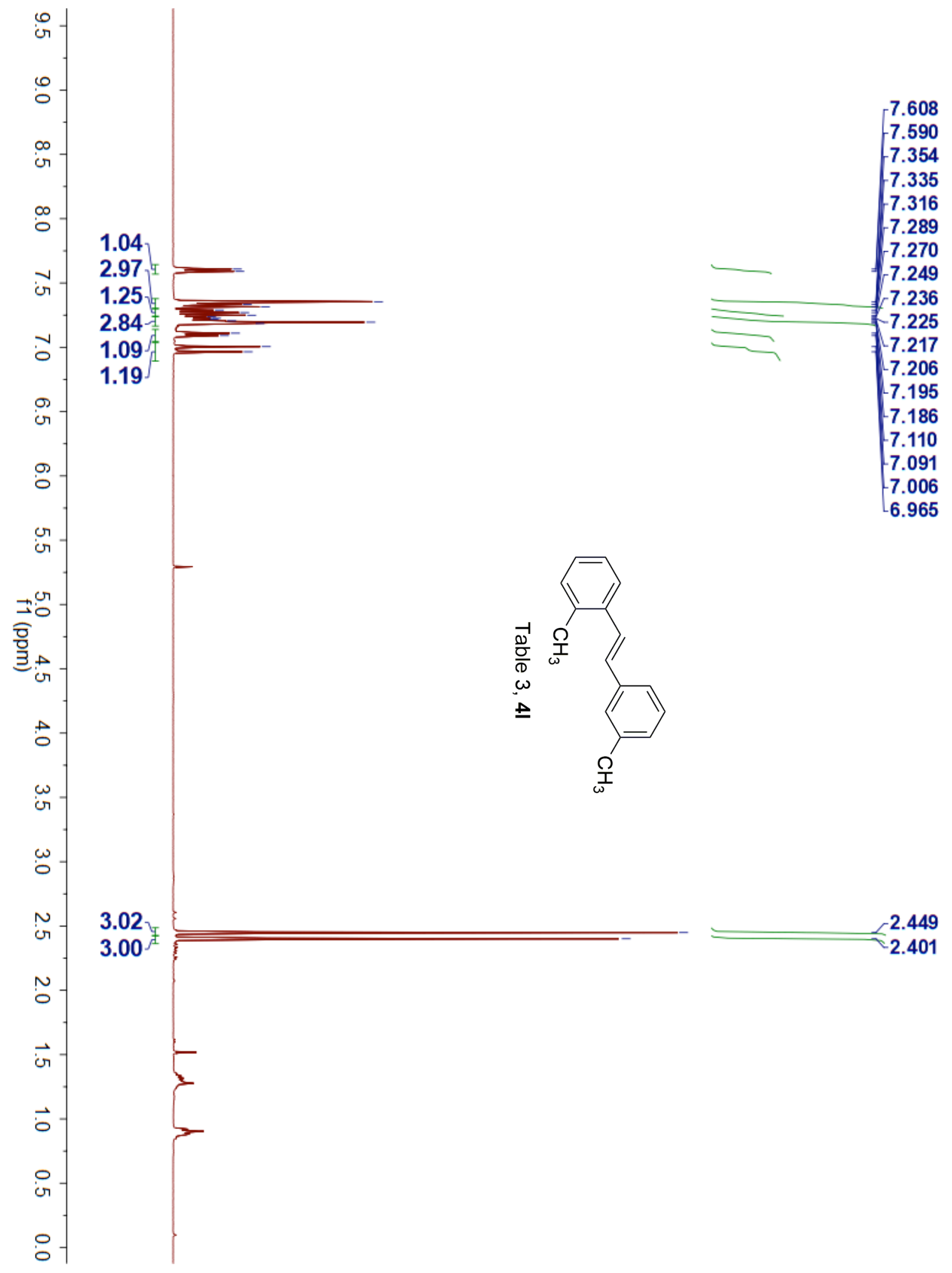




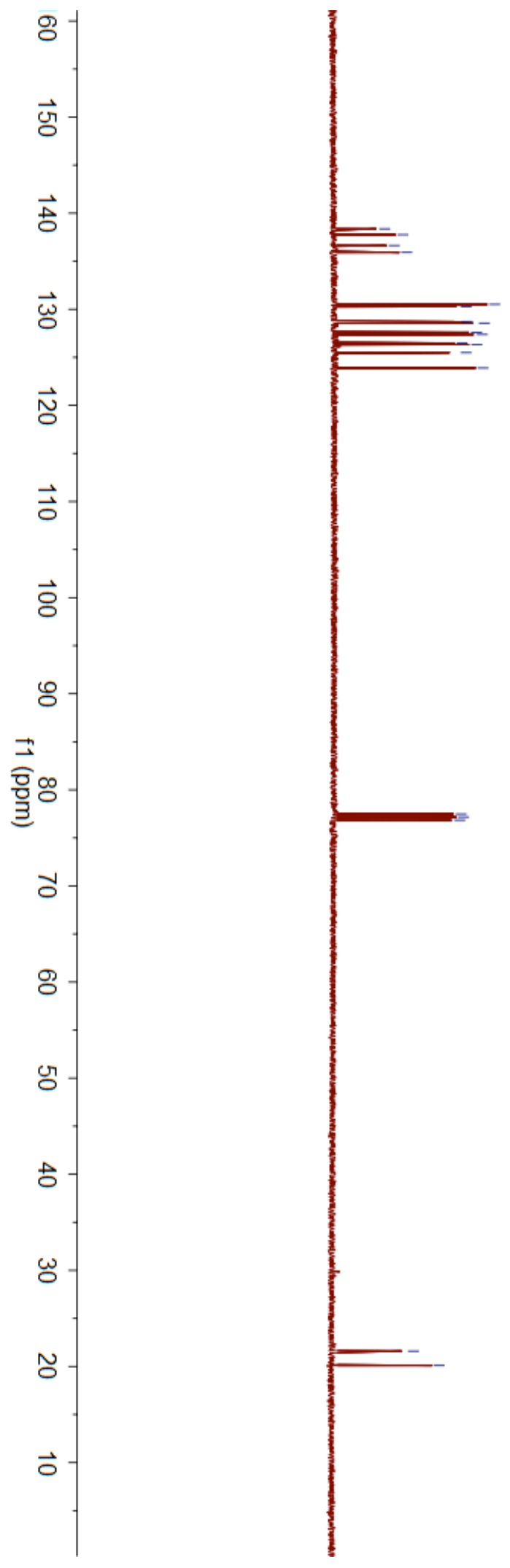

138.373

137.764

136.638

135.917

- 130.519

130.272

128.720

$-128.564$

127.611

127.379

$-126.483$

$-126.326$

$-125.489$

123.892

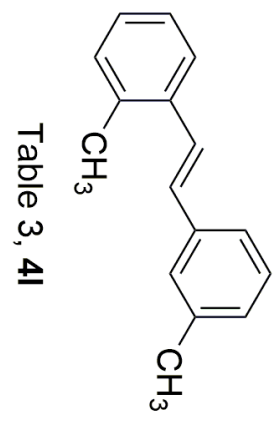

77.478

$-77.160$

$-76.843$

$-21.599$

20.098 


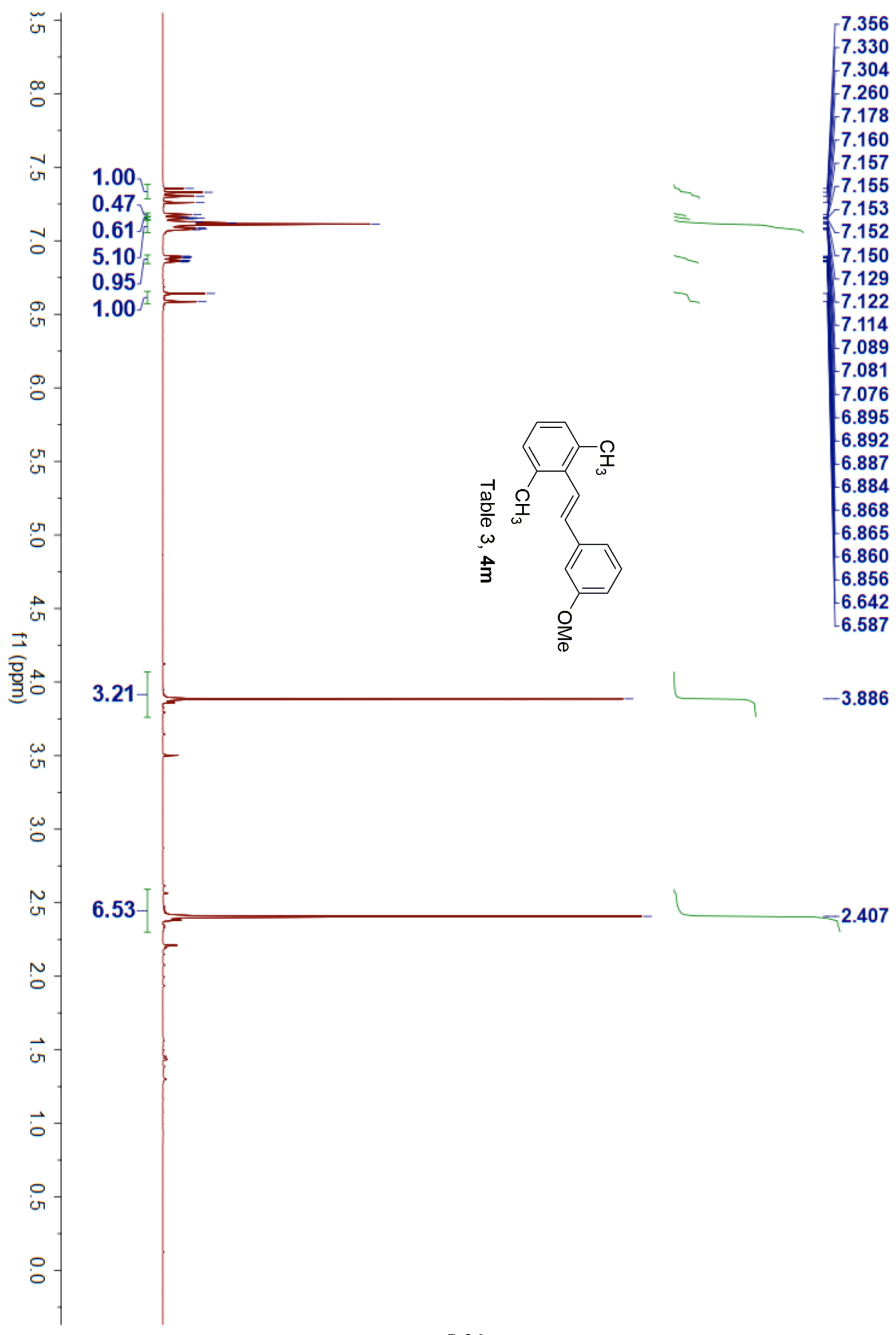




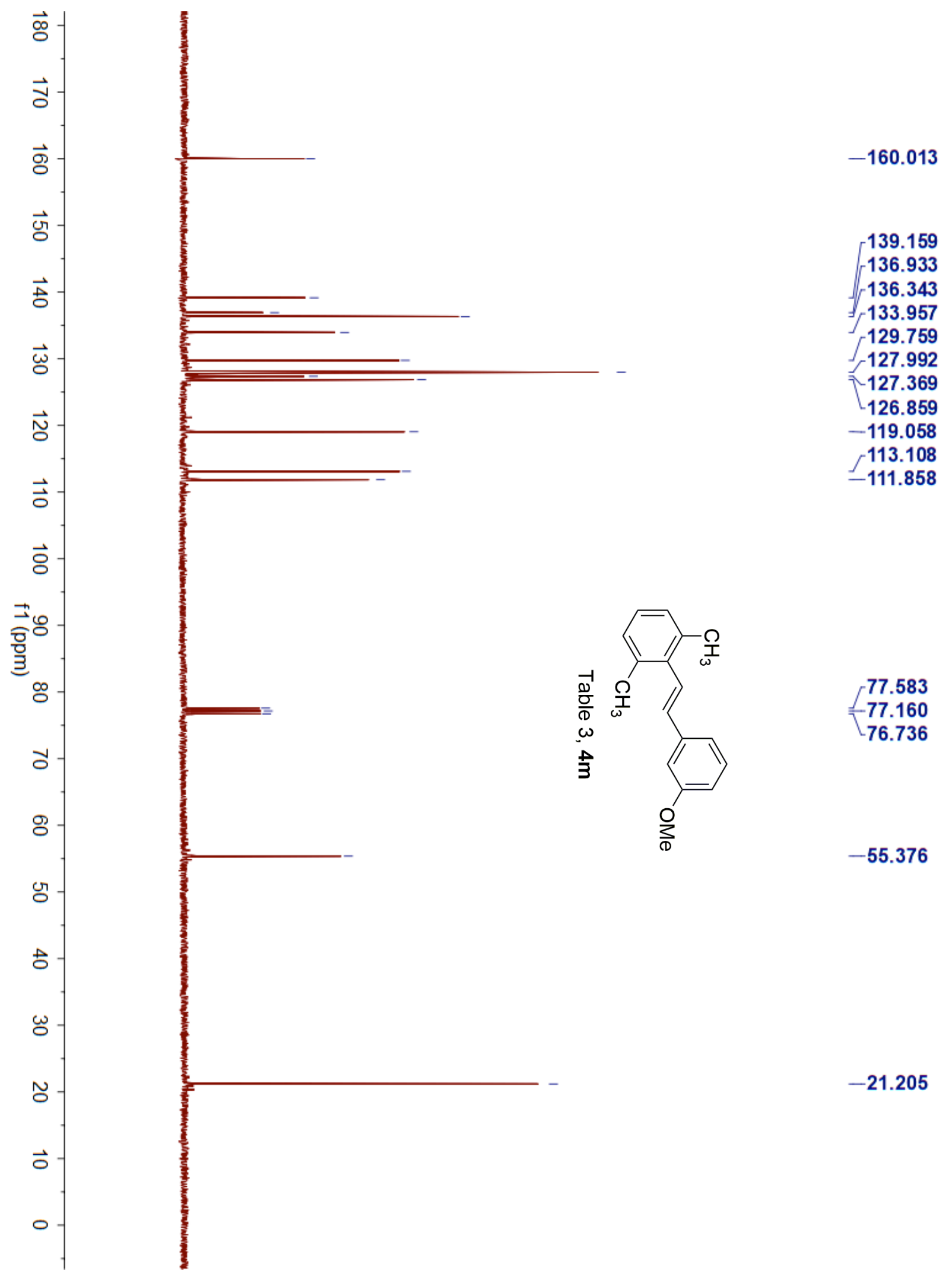




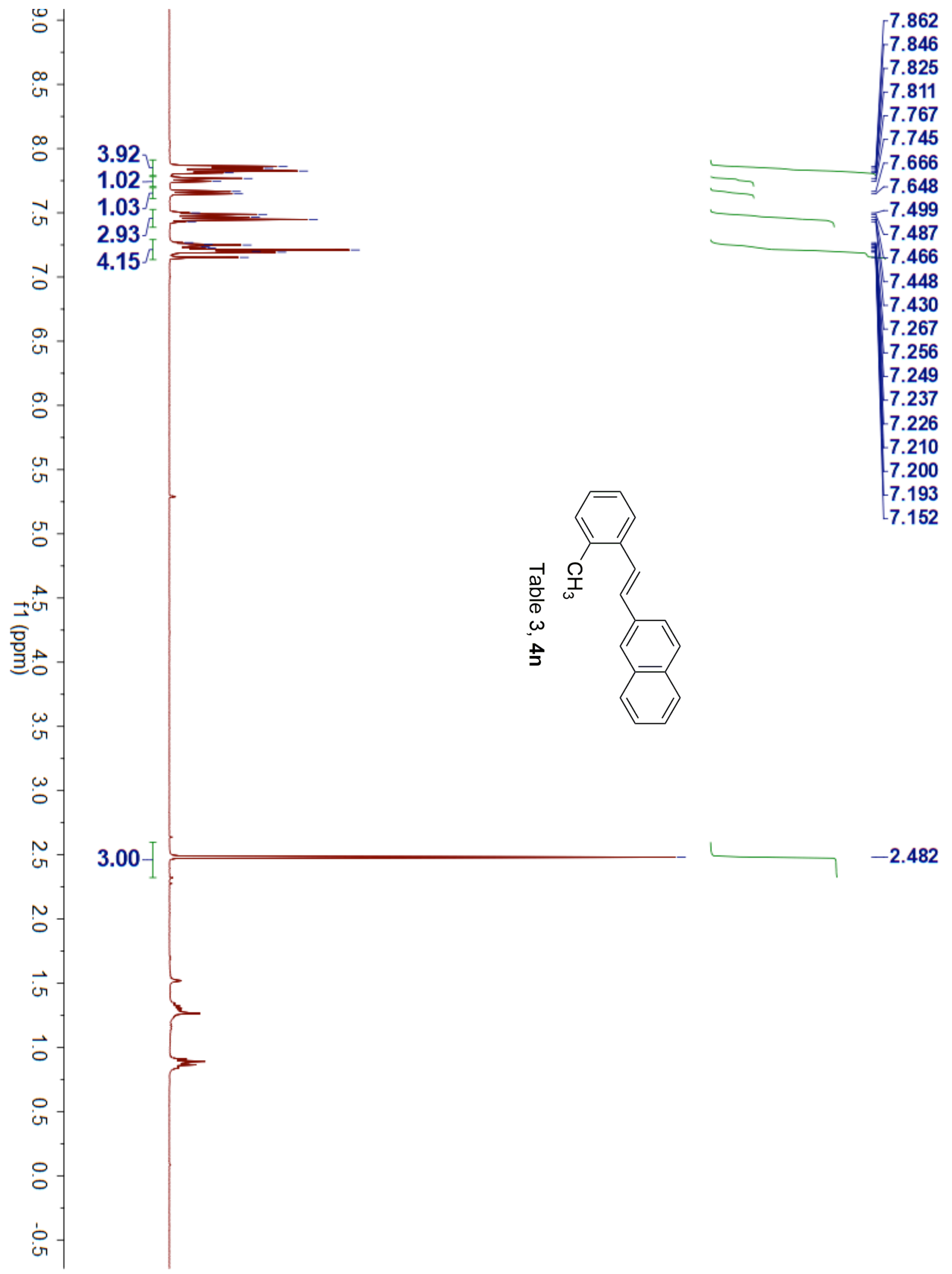




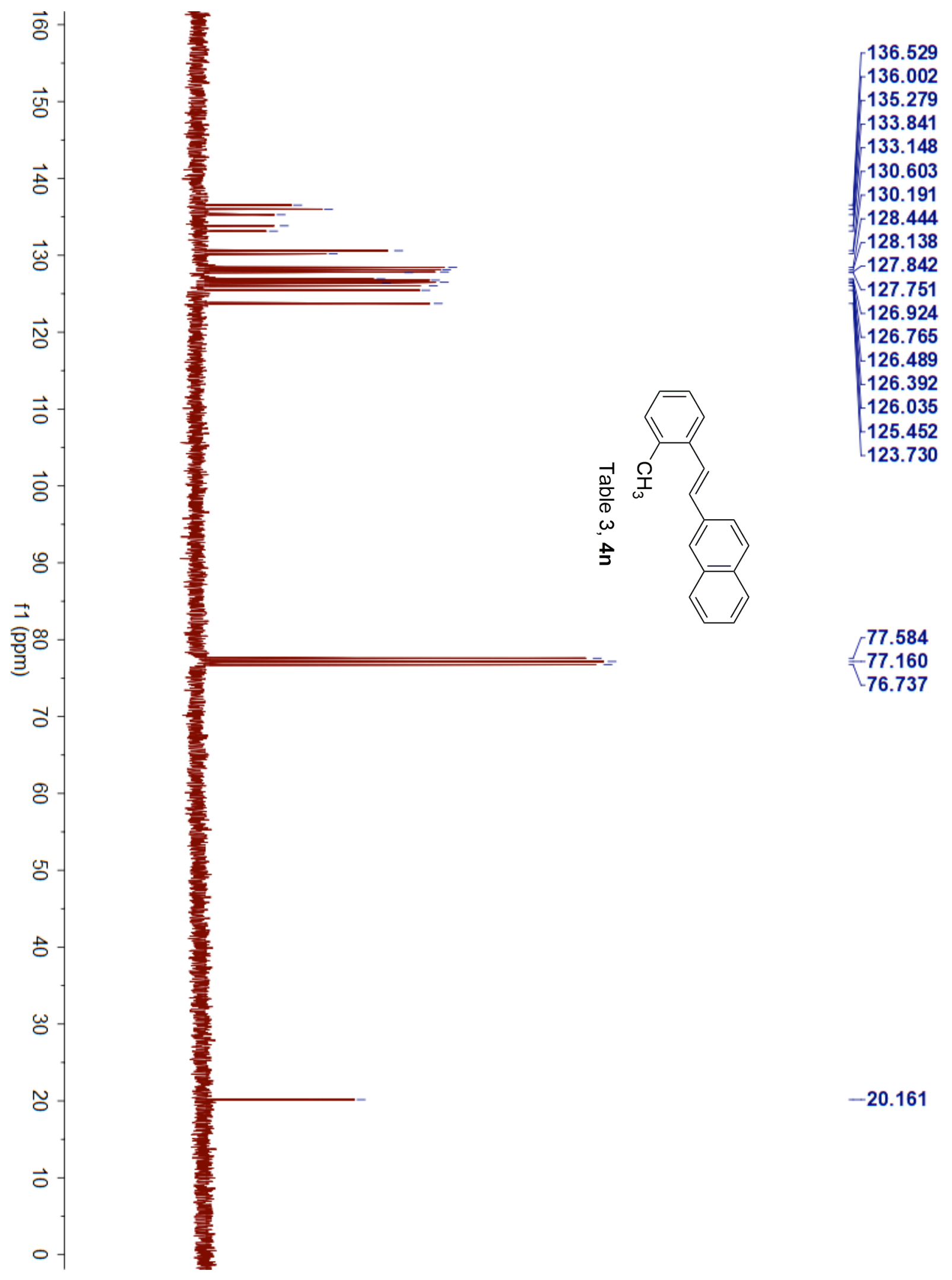




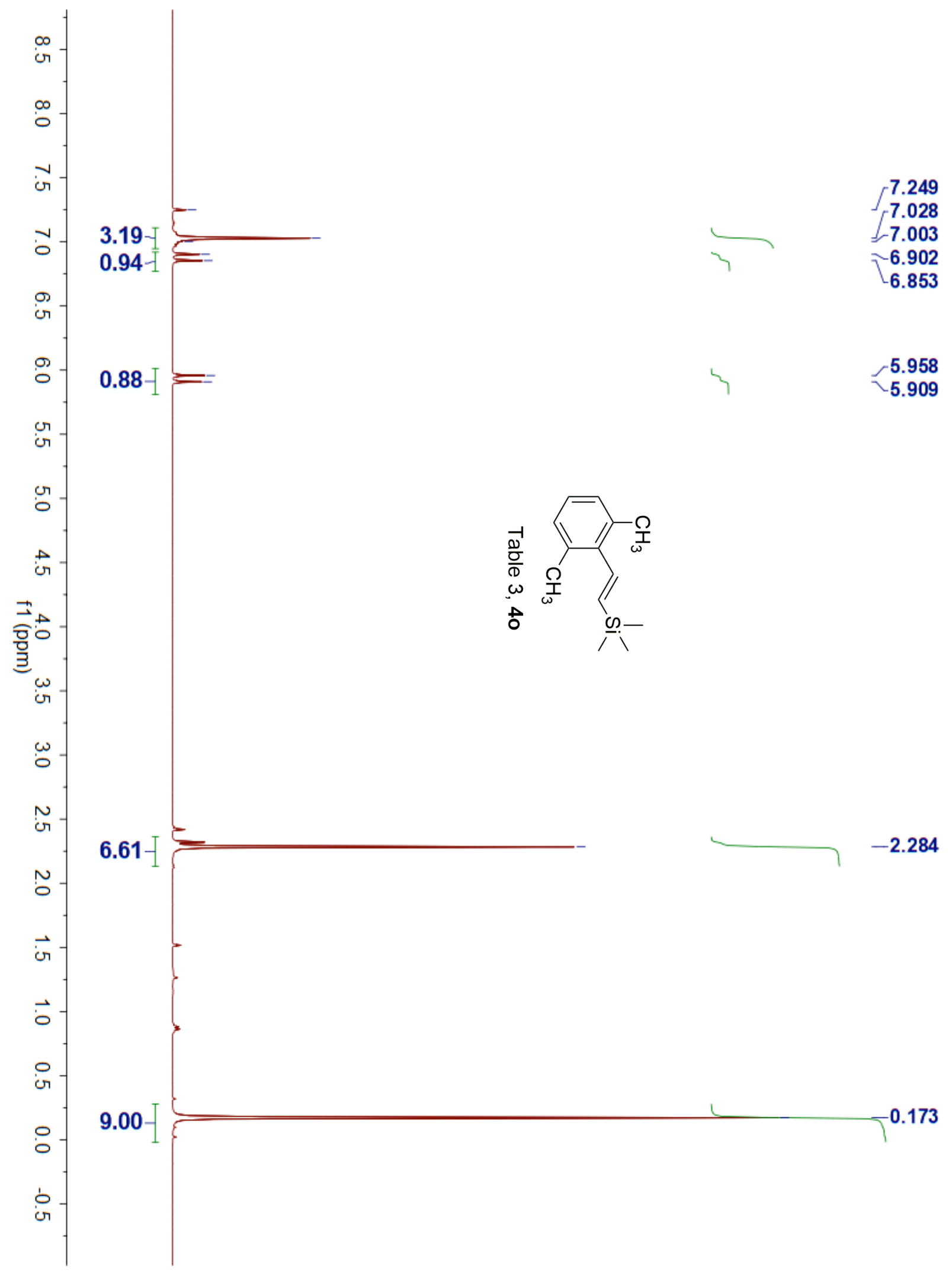




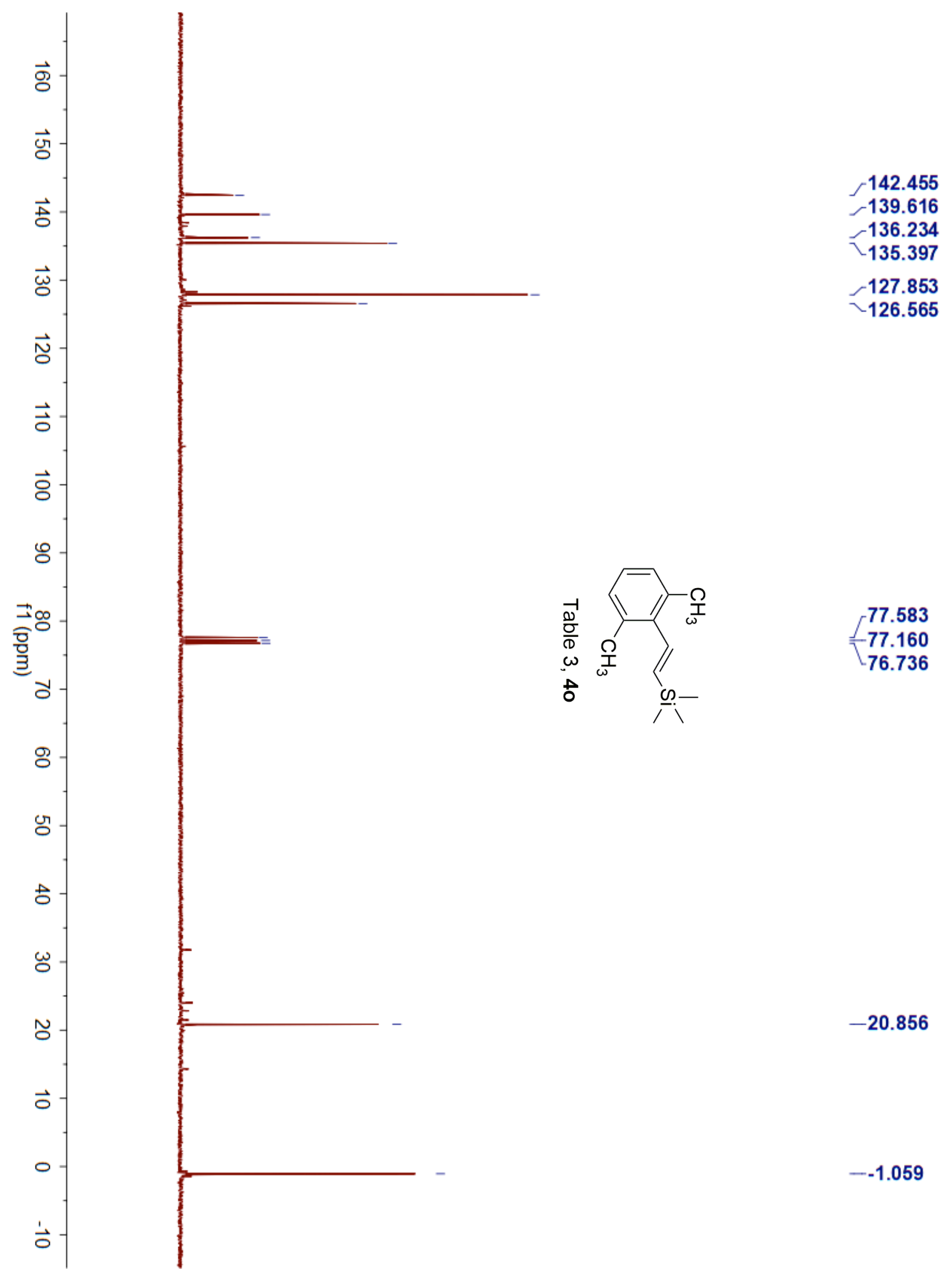




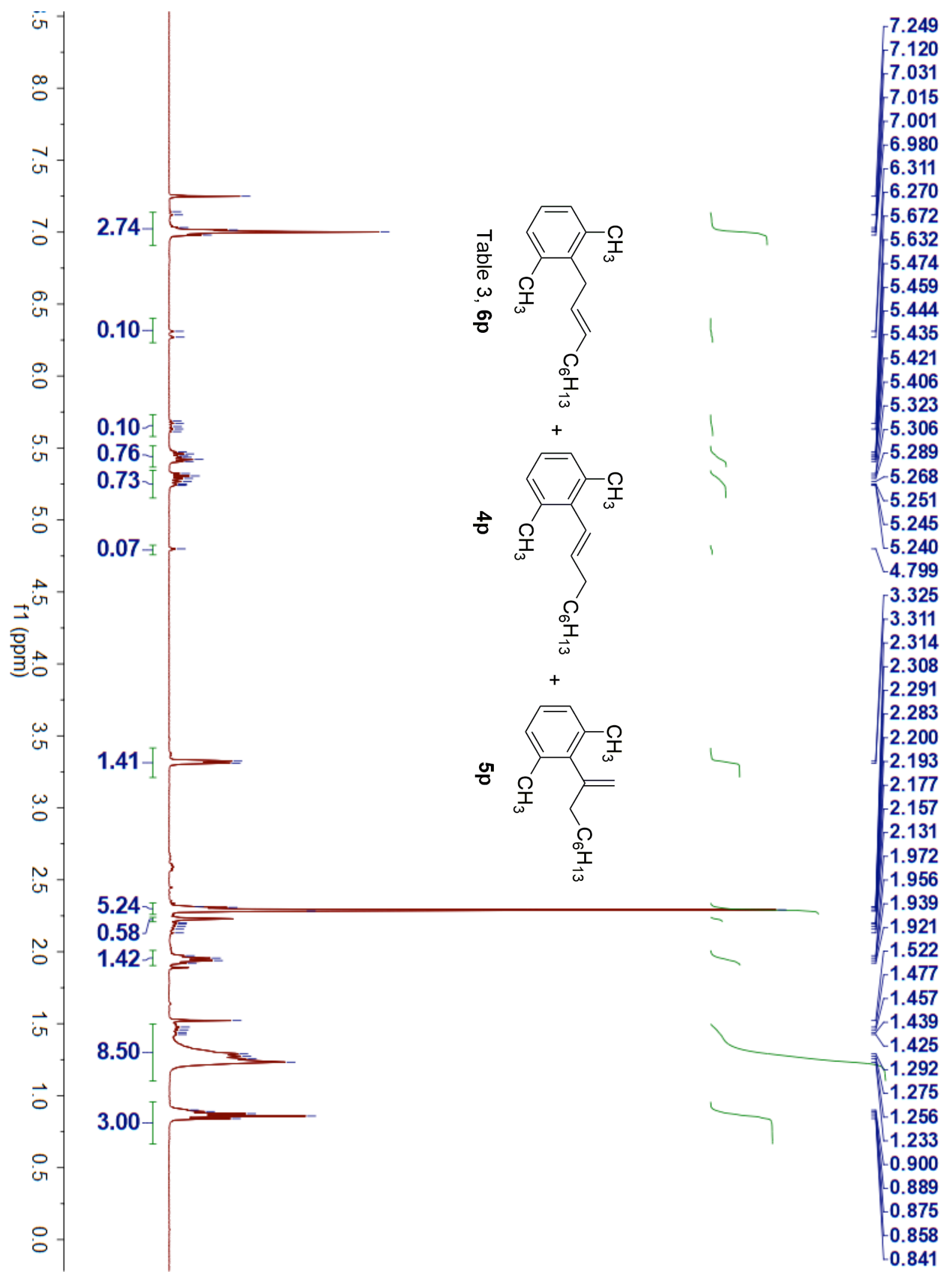




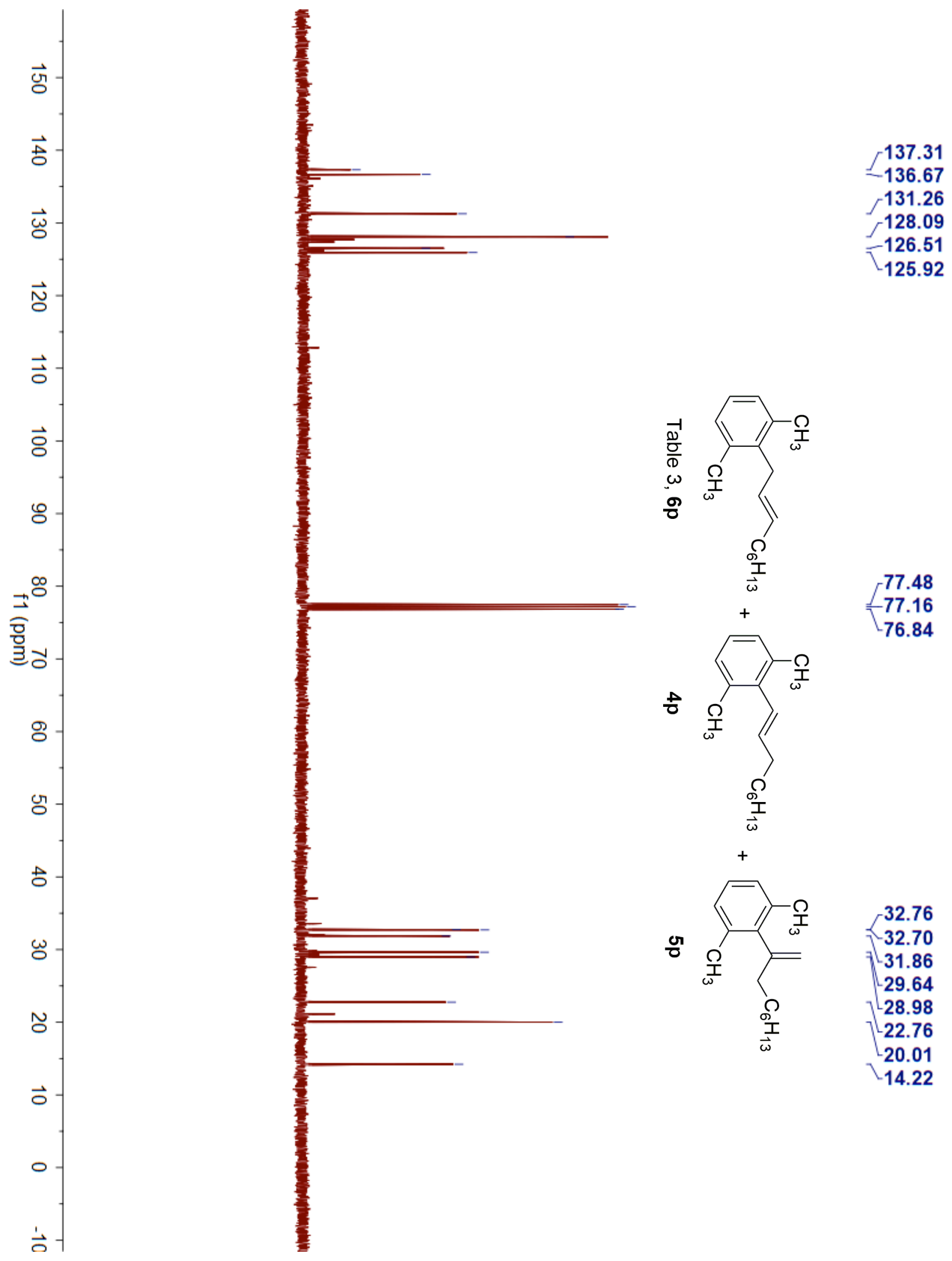




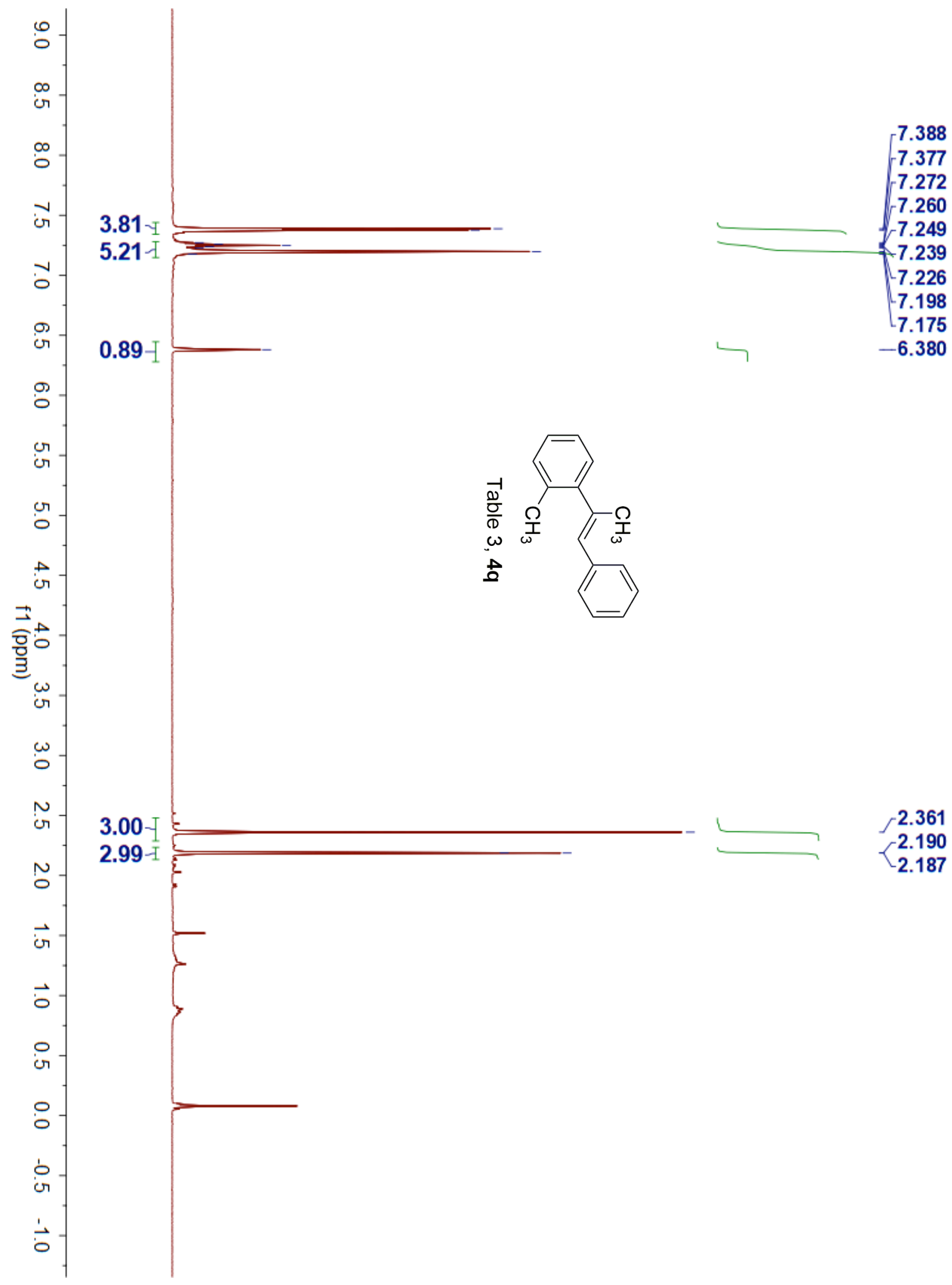




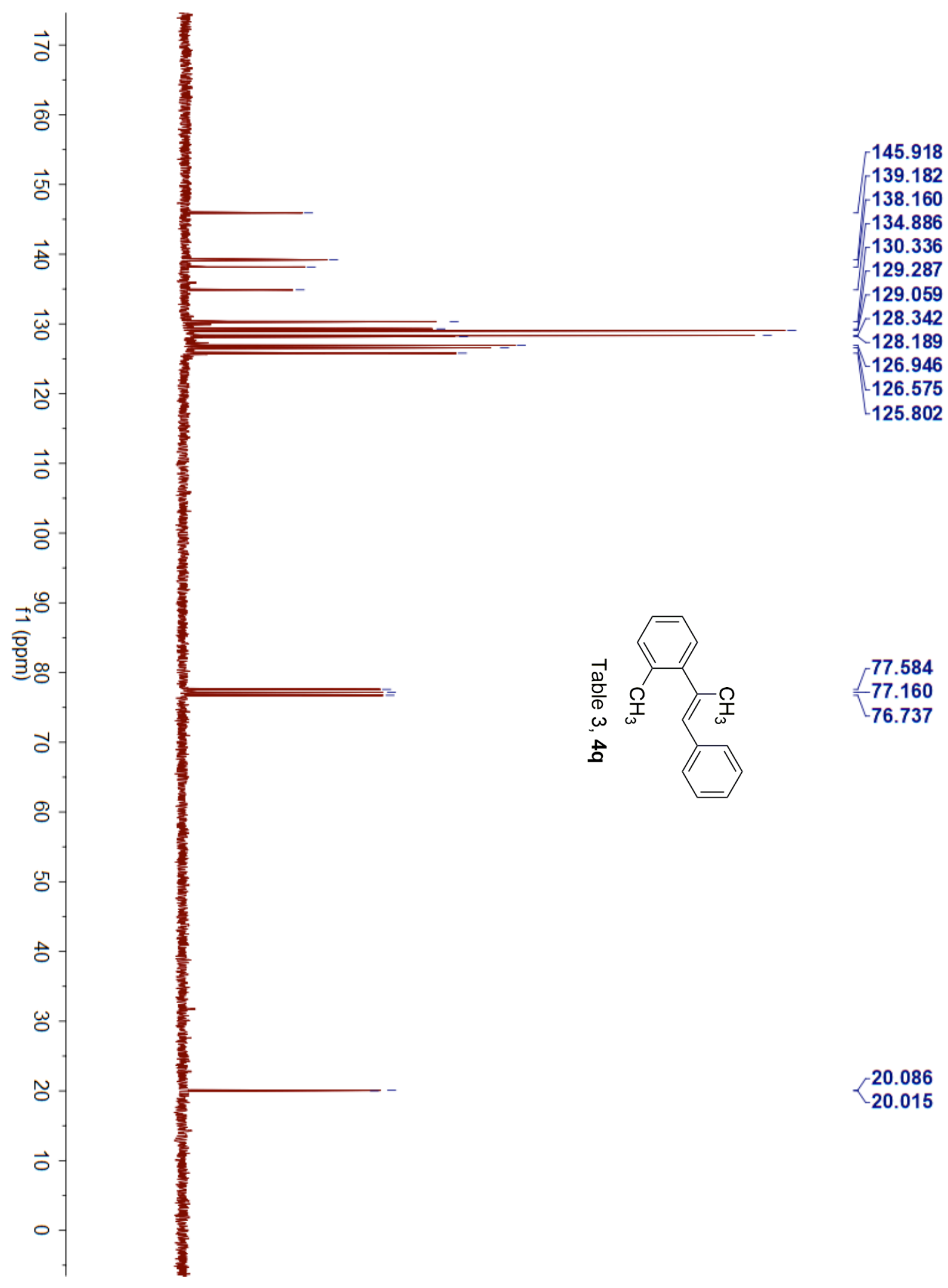




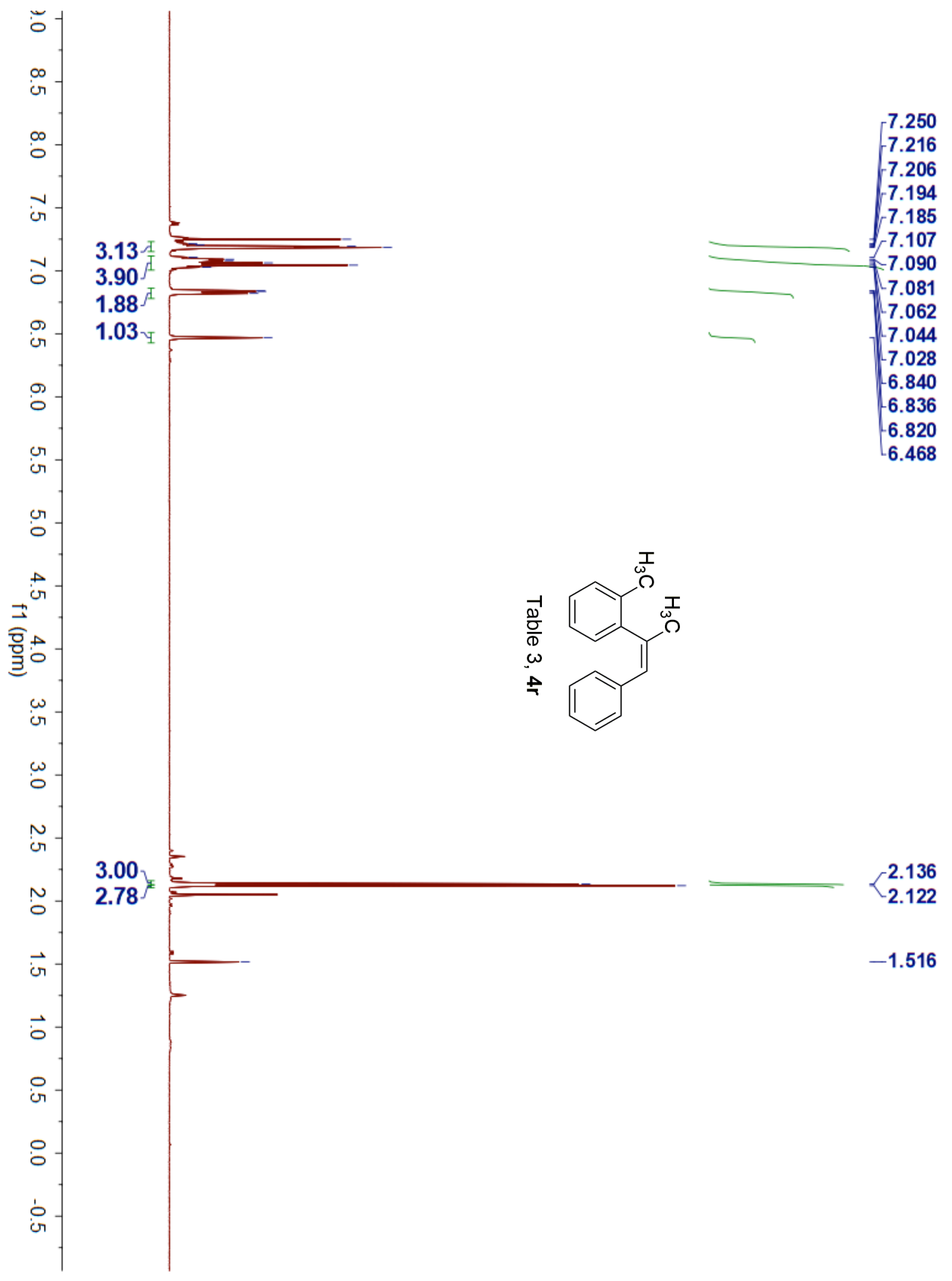




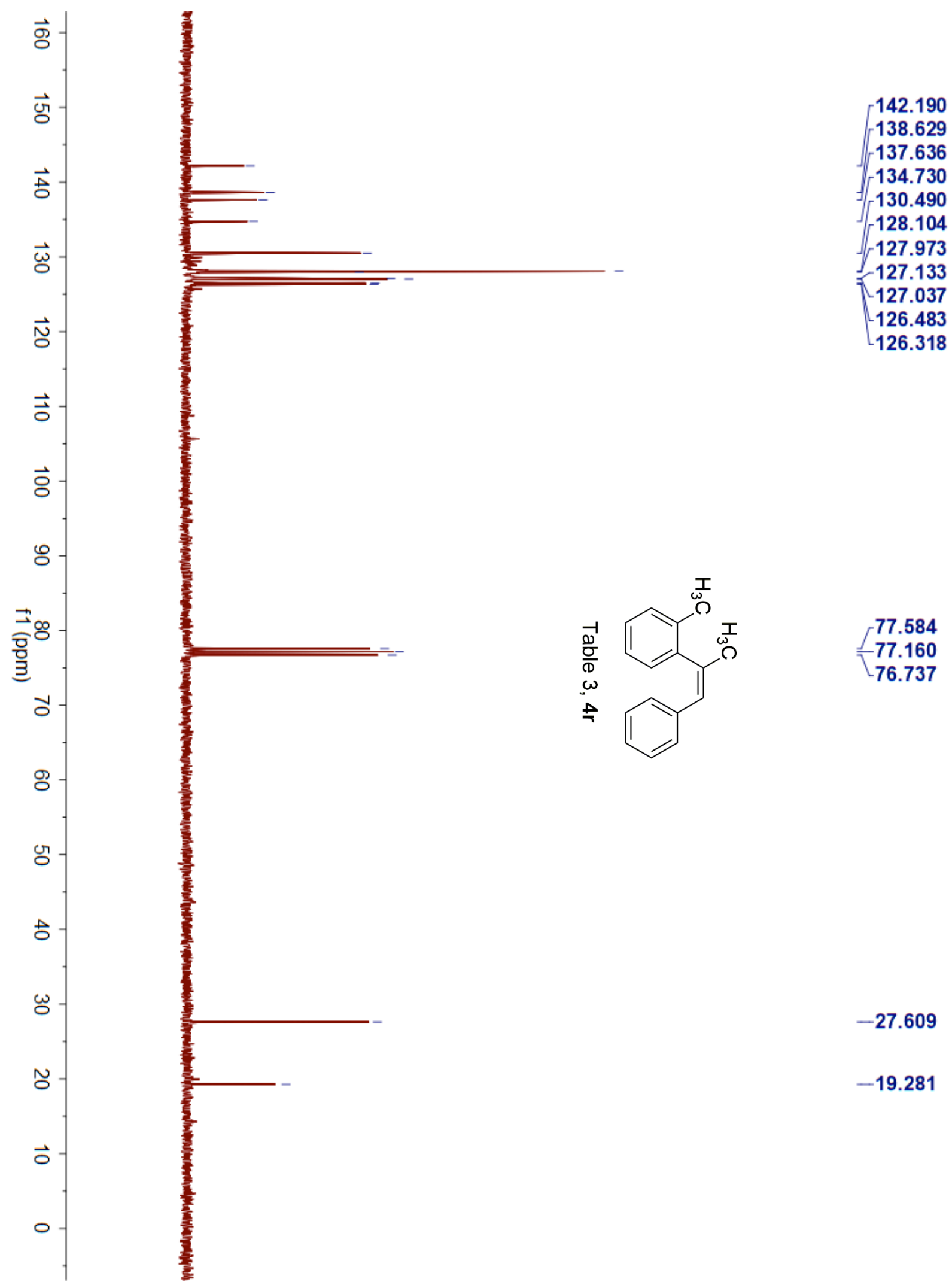




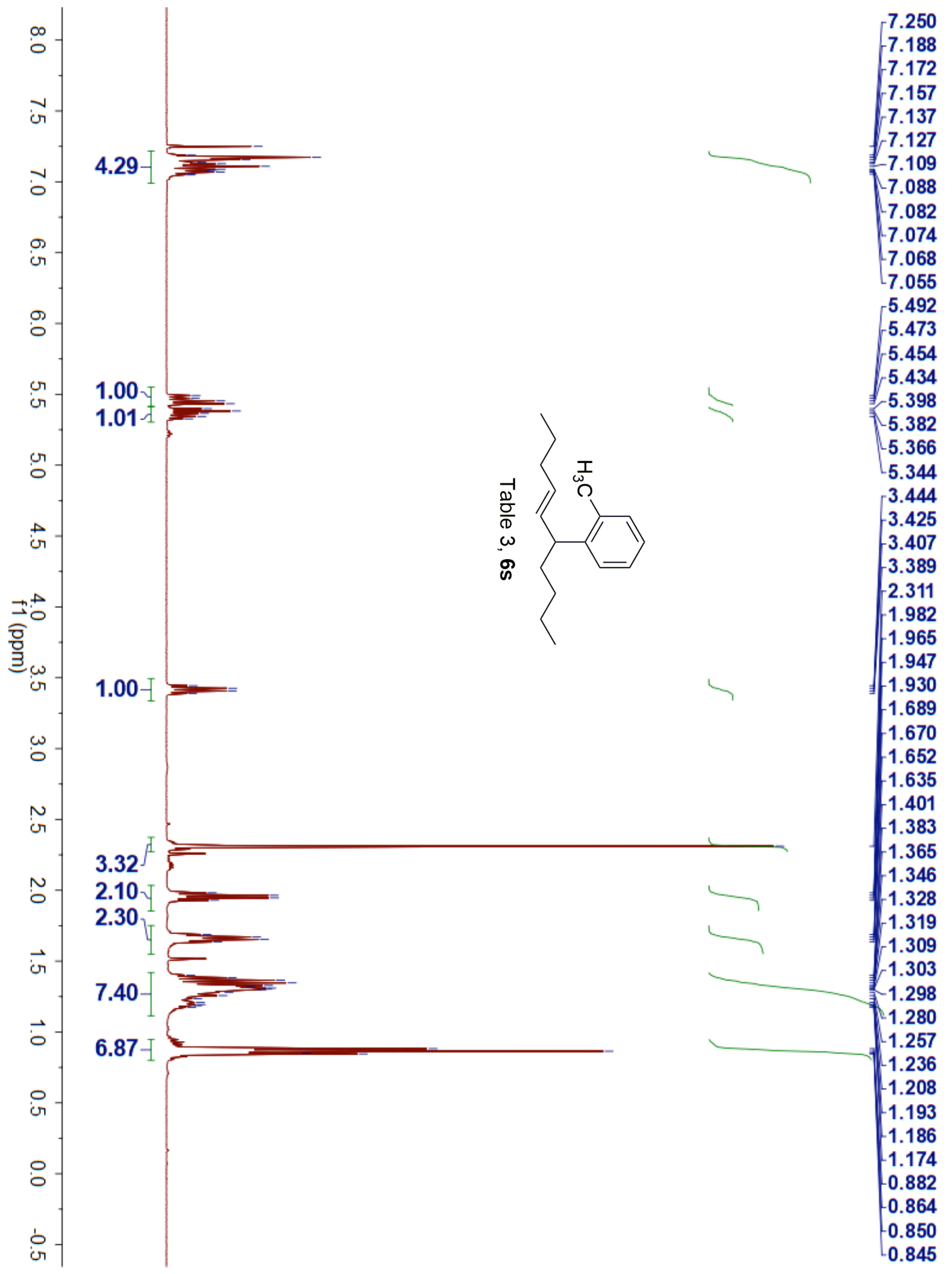



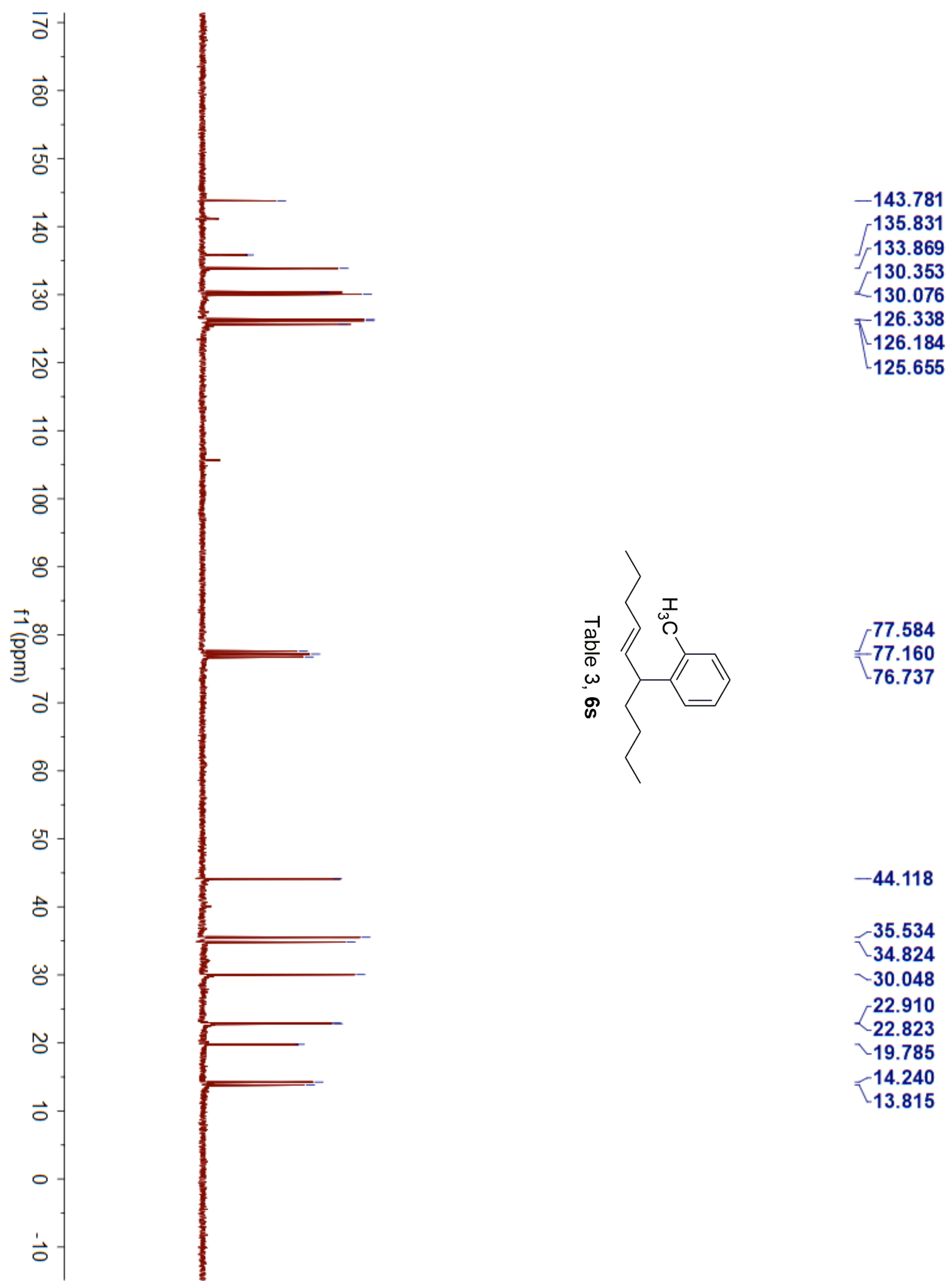

$-44.118$

$-35.534$

$-34.824$

$-30.048$

22.910

22.823

$\backslash 19.785$

$-14.240$

$-13.815$ 


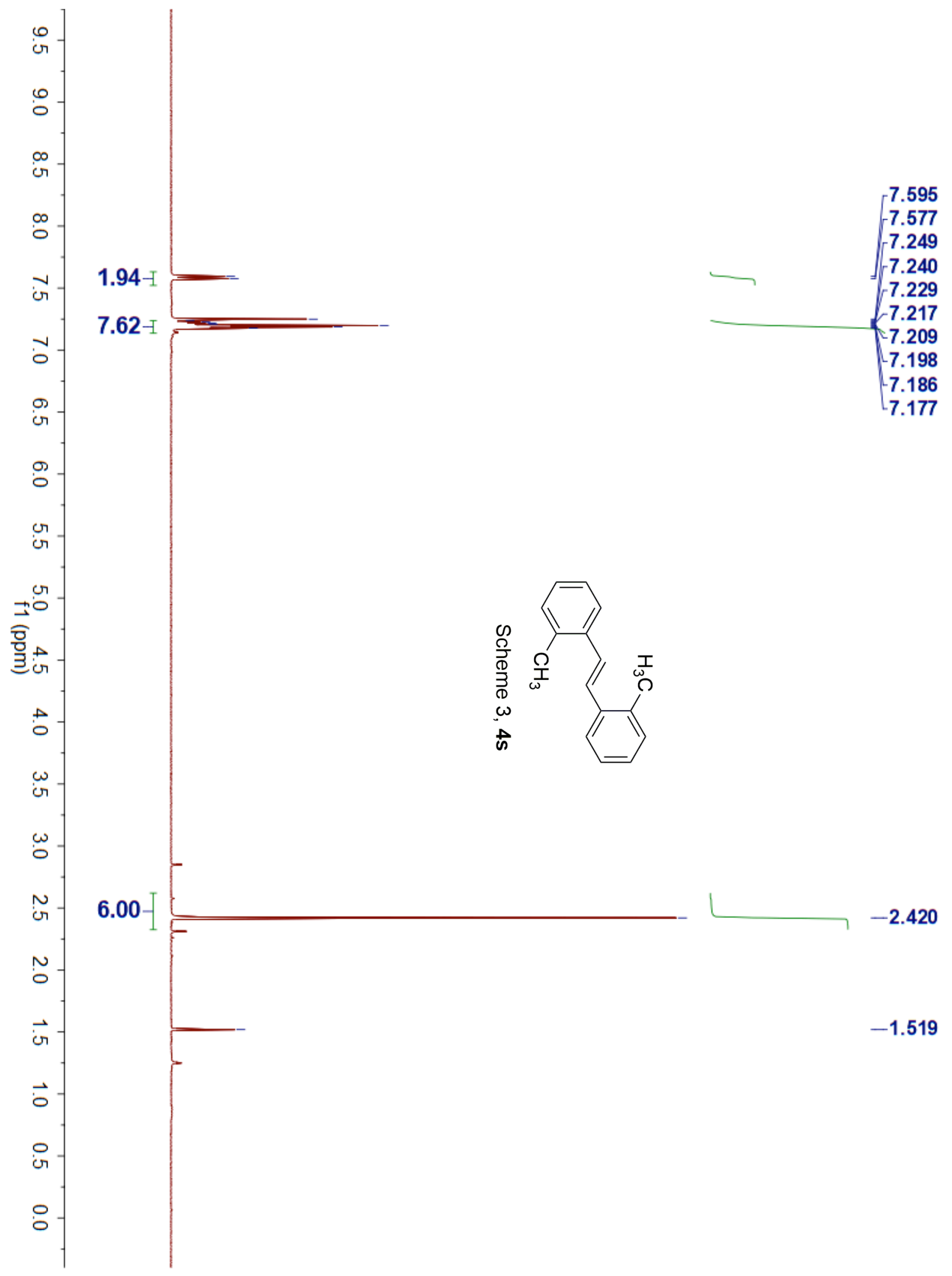




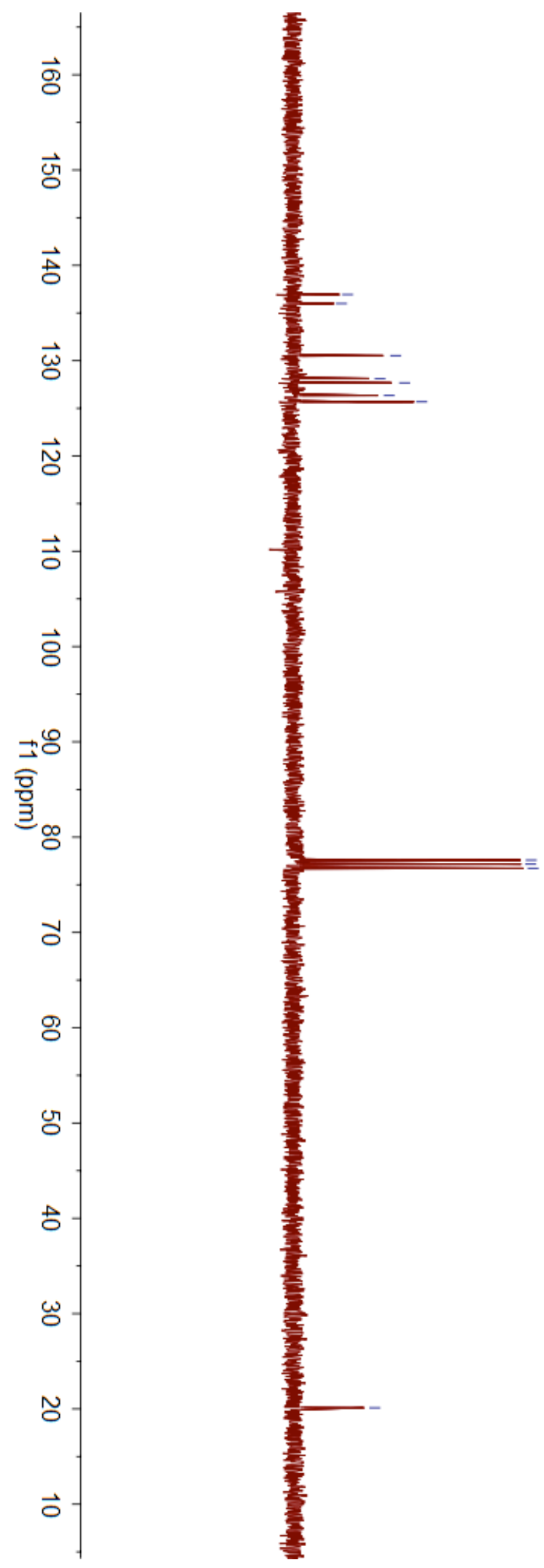

$\int_{-135.996}^{136.937}$

130.531

$\int_{-128.130}$

127.678

126.337

$-125.677$

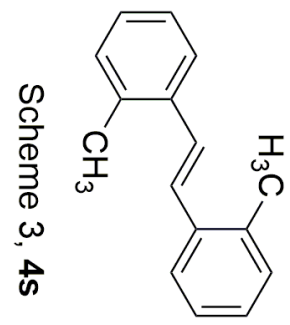

77.583
-77.160
76.736 


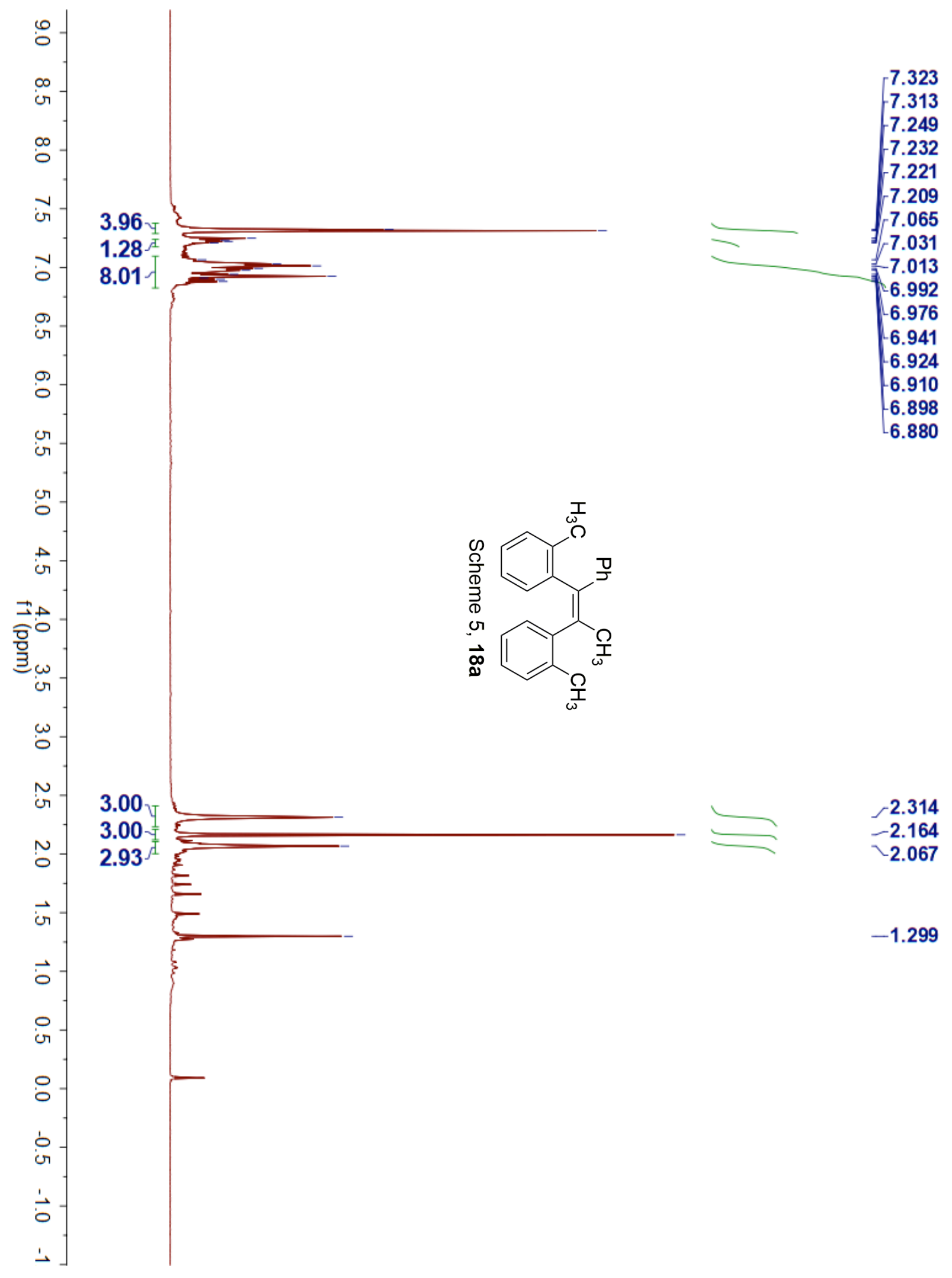




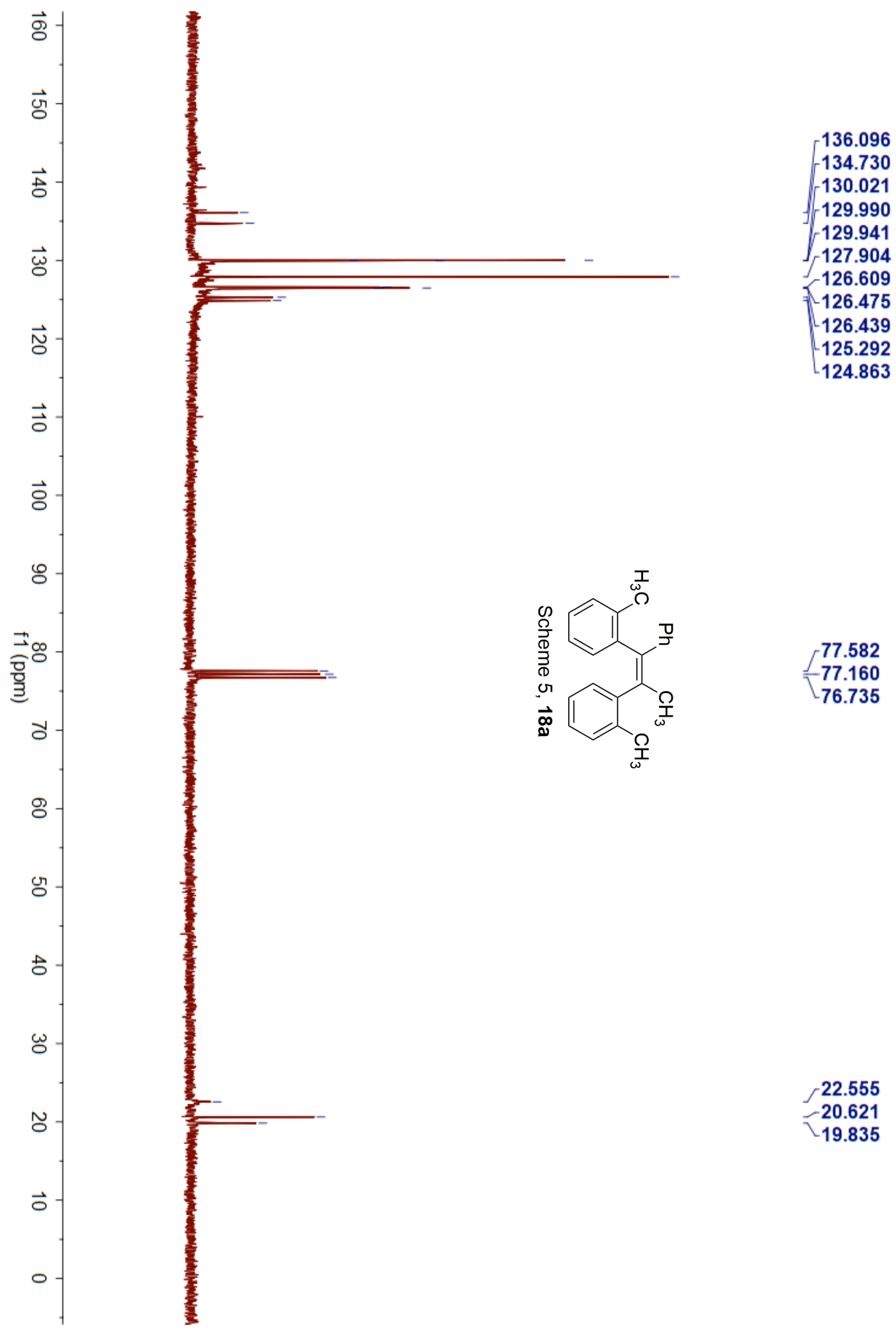




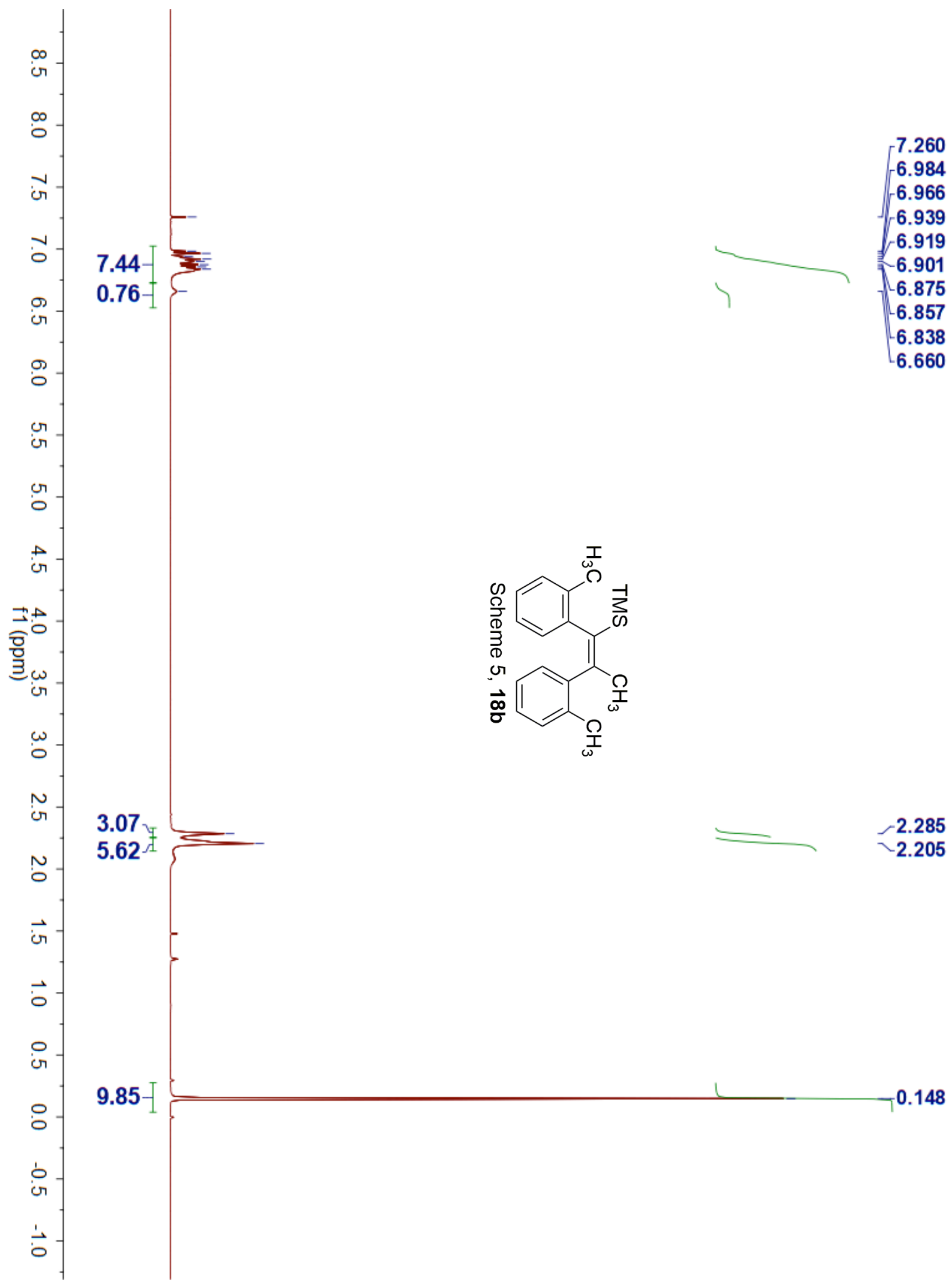




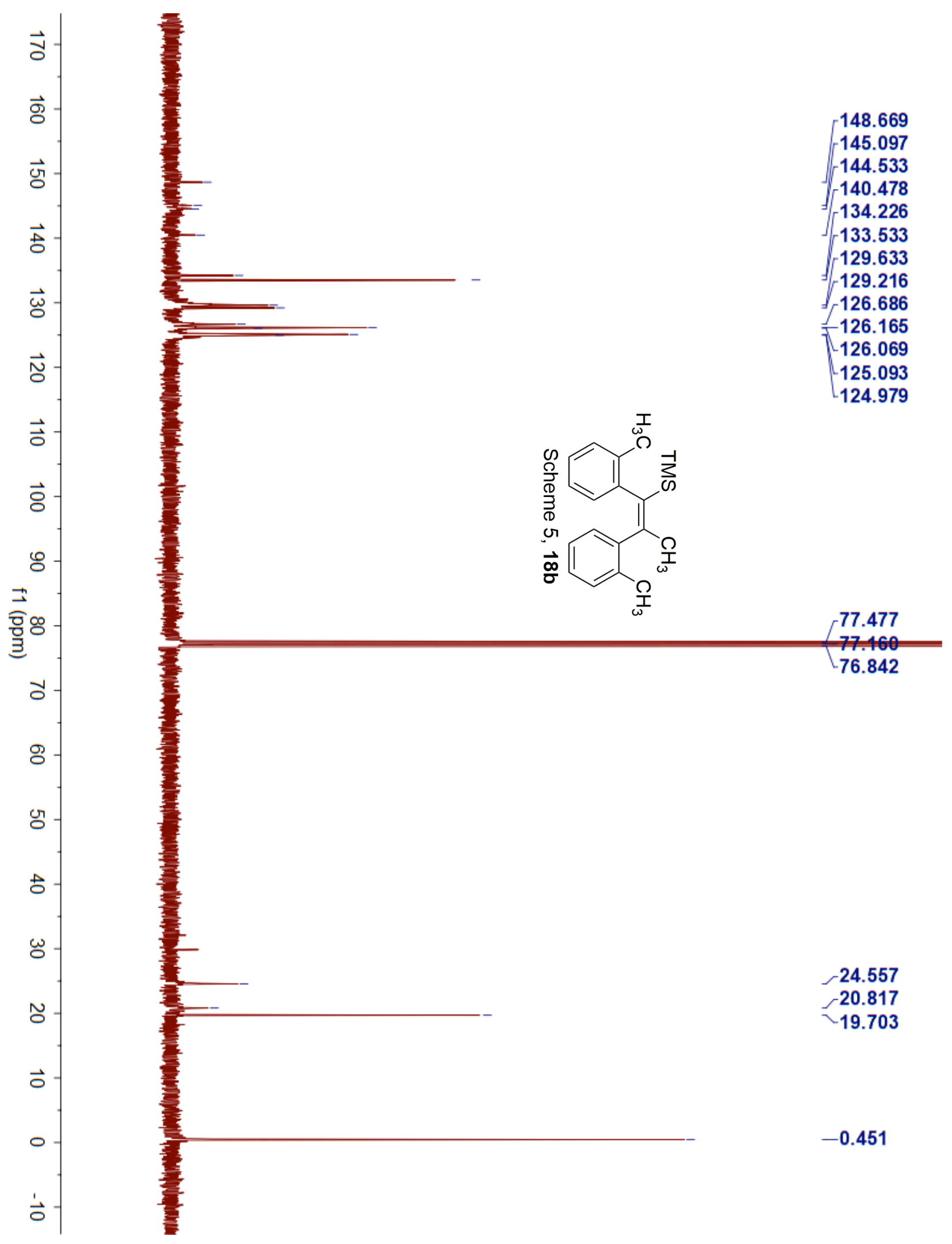

